\title{
Atomistic simulations of damage production by single and molecular ion irradiation in GaN
}

\author{
Mohammad Wali Ullah \\ Division of Materials Physics \\ Department of Physics \\ Faculty of Science \\ University of Helsinki \\ Helsinki, Finland
}

Academic Dissertation

To be presented, with the permission of the Faculty of Science of the University of Helsinki, for public criticism in auditorium XV of the Main Building of the University of Helsinki, on November 15th 2014, at 12 o'clock noon. 
ISBN 978-952-10-8969-5 (printed version)

ISSN 0356-0961

Tampere 2014

Juvenes Print

ISBN 978-952-10-8970-1 (PDF version)

http://ethesis.helsinki.fi

Helsinki 2014

Electronic Publications @ University of Helsinki 
Mohammad Wali Ullah, Atomistic simulations of damage production by single and molecular ion irradiation in GaN, University of Helsinki, 2014, 53 p. + appendices, Report Series in Physics HU-PD221, ISSN 0356-0961, ISBN 978-952-10-8969-5 (printed version), ISBN 978-952-10-8970-1 (PDF version)

\begin{abstract}
Gallium nitride $(\mathrm{GaN})$ has emerged as one of the most important semiconductors in modern technology. GaN-based device technology was mainly pushed forward by invention of p-type doping and the successful fabrication of light emitting diodes (LEDs) and laser diodes (LDs). Intensive studies in the last 20 years on GaN have significantly advanced the understanding of the properties and have expanded the range of practical applications. Beside basic lighting, current applications of GaN include high-power and high temperature electronics, microwave, optoelectronic devices, and so on.
\end{abstract}

The successful production of optical devices demands efficient tuning of charge carrier lifetime where defect engineering plays a vital role. During growth, varying the level of recombination centers is difficult, whereas ion irradiation can do this job efficiently on a final product. On the other hand, during doping, undesirable defects can also be produced and epitaxial $\mathrm{GaN}$ is known to have a highly defective structure. Thus, having both positive and negative aspects, it is very important to have a detailed understanding of irradiation-induced defects.

To explain experimental findings, atomic level understanding is necessary, but it is not always possible to have an atomistic view of defect dynamics in experiments. Some damage build-up studies by single ions have been reported in the literature, but not many by molecular ions. In this thesis, the irradiation of $\mathrm{GaN}$ by single and molecular ions by the means of atomistic simulations was studied. Detailed analysis mainly of what kind of defects, their distribution, reason of defect formation and time evolution have been studied and compared with experiments.

The irradiation response of both bulk and nano-structured GaN system were studied. For bulk studies, all projectiles were irradiated having the same energy per mass. The damage by molecular ions showed strong dynamic annealing. No non-linearity had been observed in the total number of point defects between single and molecular ions. On the other hand, molecular ions produce larger clusters of point defects than single ions. These large defect clusters can be one of the mechanisms of the experimentally observed faster carrier decay time for molecular projectiles. Defects were mostly concentrated at the surface and near-surface regions, which is also evident from experiments. 
Comparison between a similar mass single ion and a molecular ion show that a single ion produced more defect clusters than molecular ions. This suggests that heavy ions are even more efficient than similar mass cluster ions to quench the carrier lifetime.

Irradiation of a $\mathrm{GaN}$ nanowire (NW) reveals that a large surface to volume ratio promotes high density of surface defects. The experimentally observed yellow luminescence band is correlated with these defect induced surface states. Irradiation induced defects also expand the lattice parameters of the NW. 


\section{Contents}

\begin{tabular}{lll}
\hline Abstract & i
\end{tabular}

Contents iii

\begin{tabular}{llr}
\hline & Introduction & 1
\end{tabular}

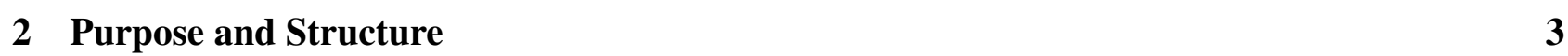

$2.1 \quad$ Summary of original publications $\ldots \ldots \ldots \ldots$

2.2 Author's own contributions $\ldots \ldots \ldots \ldots \ldots \ldots$

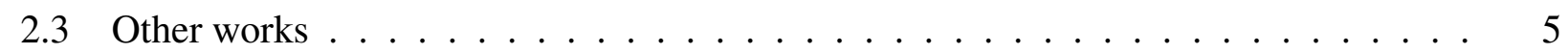

\begin{tabular}{lll}
\hline 3 & Gallium Nitride & 7
\end{tabular}

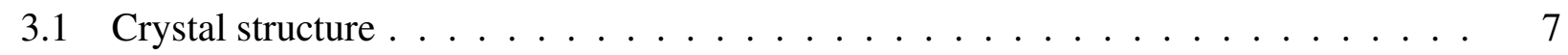

$3.1 .1 \quad$ Wurtzite . . . . . . . . . . . . . . . . . . 7

3.2 GaN bulk crystal growth $\ldots \ldots \ldots \ldots \ldots$

3.2.1 High pressure nitrogen solution growth process . . . . . . . . . . . . 9

3.2 .2 Ammonothermal method . . . . . . . . . . . . . . . . . . . 10

3.2 .3 Na flux growth technology $\ldots \ldots \ldots \ldots \ldots \ldots$

3.3 Applications $\ldots \ldots \ldots \ldots \ldots \ldots \ldots \ldots \ldots$ 
3.4 Optical properties . . . . . . . . . . . . . . . . . 12

\begin{tabular}{|lr}
\hline 4 Ion Irradiation & 14
\end{tabular}

$4.1 \quad$ Stopping power and range $\ldots \ldots \ldots \ldots \ldots \ldots$

4.2 Effects of ion irradiation on materials $\ldots \ldots \ldots \ldots$

$4.2 .1 \quad$ Point and cluster defects $\ldots \ldots \ldots \ldots \ldots \ldots \ldots$

4.3 Ion irradiation of $\mathrm{GaN} \ldots \ldots \ldots \ldots \ldots \ldots$

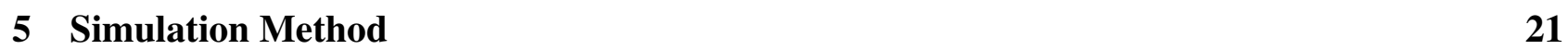

$5.1 \quad$ Molecular dynamics $\ldots \ldots \ldots \ldots \ldots \ldots \ldots \ldots$

5.2 Interatomic potentials $\ldots \ldots \ldots \ldots \ldots \ldots \ldots \ldots$

$5.2 .1 \quad$ Analytical bond-order potentials . . . . . . . . . . . . . . 25

5.2 .2 Energetic short-range interaction $\ldots \ldots \ldots \ldots \ldots$

5.2 .3 Lennard-Jones potential $\ldots \ldots \ldots \ldots \ldots \ldots$

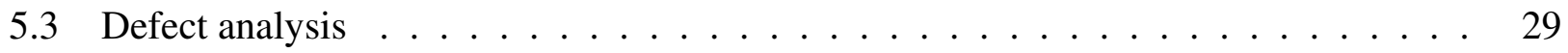

\begin{tabular}{|lrl}
\hline 6 & Results & 30
\end{tabular}

6.1 Comparative study of atomic and molecular projectiles . . . . . . . . . . 30

6.2 High energy irradiation and defect clustering $\ldots \ldots \ldots 33$

6.3 Mass effect on defect formation $\ldots \ldots \ldots \ldots$

6.4 Irradiation of a GaN nanowire $\ldots \ldots \ldots \ldots \ldots$

\begin{tabular}{lll}
\hline 7 & Conclusions & 42
\end{tabular}

\begin{tabular}{ll}
\hline Bibliography & 46
\end{tabular} 


\section{Chapter 1}

\section{Introduction}

Semiconductor materials have been studied intensively since the birth of silicon ( $\mathrm{Si}$ ) technology. A key factor for the multitude of electronic devices in our life is the ability to physically and chemically tailor the properties of semiconductors. Semiconductor materials like Si and GaAs have been extensively studied and have found many applications in different fields. But there are fundamental property limitations, for instance, in power electronic devices Si technology has already touched its limit. In response to these limitations, devices based on high band gap materials like GaN are being developed.

Gallium Nitride $(\mathrm{GaN})$ was first synthesized in 1932 by Johnson et al. [1]. There was a major boost in the $\mathrm{GaN}$ research around 1970 [2] but failure of producing p-type material faded most of the activities. In late 1980s, the research on $\mathrm{GaN}$ and related materials got new life after Akasaki et al. produced smooth single crystal GaN on a foreign substrate and fabricated the world's first GaN p-n junction UV-light-emitting diode (LED) [3-5]. Based on this, in 1994 Nakamura et al. demonstrated the first high brightness blue LED [6]. Since then, much has been done in the development of better and more efficient GaN-based devices.

In recent years, group III-nitrides have become third in importance after Si and GaAs among semiconductor materials systems and are rapidly moving to overtake GaAs [7]. Figure 1.1 shows some dominating properties of $\mathrm{GaN}$ over $\mathrm{Si}$ and $\mathrm{SiC}$ which make it a strong candidate for high power applications. The chemical stability at high temperatures combined with the significant hardness make $\mathrm{GaN}$ an important material for protective coatings. By alloying $\mathrm{GaN}$ with $\mathrm{AlN}$ and $\mathrm{InN}$ a continuous range of direct band gap energies from the visible spectrum to the UV wavelengths can be spanned. This makes the nitride system attractive for optoelectronic device applications, such as LEDs, lasers, and detectors, which are active in the green, blue or UV wavelengths [8]-10]. 


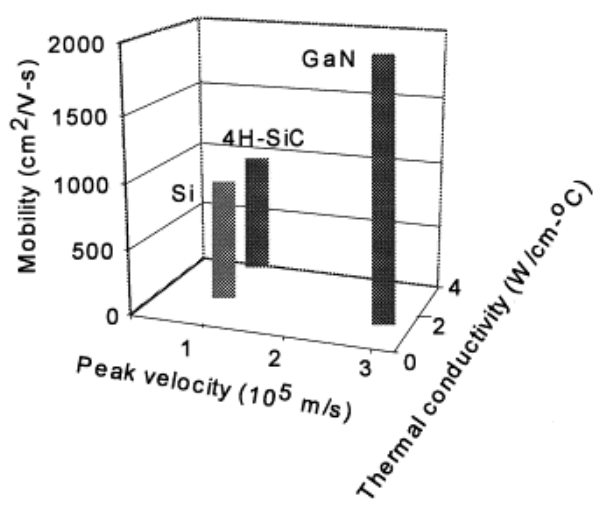

Figure 1.1: Comparison between properties of $\mathrm{Si}, \mathrm{SiC}$ and $\mathrm{GaN}[9]$.

In the fabrication of $\mathrm{GaN}$ based devices, effective and controlled doping is essential to modify the electrical and optical properties. Ion implantation is one of the important players for doping of semiconductor materials. GaN inherently possesses a high defect density and techniques like ion irradiation produce more defects in the material. To get a high quality product, a low defect density is desirable, at the same time in some applications defects can be used in a positive way. For instance, it is possible to introduce controlled defects and impurities which are optically active [11]. Thus, ion irradiation offers wide possibilities of defect engineering through the choice of implantation species, dose, and energy. It is well-known that a successful application of ion implantation depends on understanding the production and annealing of radiation damage. Thus, detailed studies of implantation damage in $\mathrm{GaN}$ are very important for further development and efficiency of applications.

The effects of single ion irradiation on defect formation of $\mathrm{GaN}$ have been studied widely. However, not many works have been focused on the defect formation behaviour when irradiation is done by molecular projectiles. Some experiments have shown that the damage production itself in semiconductors is non-linear with the deposited energy density of single and molecular projectiles [12-14]. There are also experiments investigating the effect of ion-beam-produced defects on luminescence properties [15-18]. Although such studies exist, atomistic level information on the dynamics and characteristics of irradiation damage is difficult to obtain from experiment. Molecular dynamics (MD) simulations [19, 20] give a theoretical description of the dynamics of the system up to the microsecond time scale at the atomic level. It is used in many fields of science like materials science, biophysics, biochemistry etc.

In this thesis, MD simulation is used to study the defect formation in GaN by single and molecular ion irradiation. Point defects and defect clusters are studied in the bulk phase of GaN as well as in a one-dimensional nanowire. All results were compared with experimental findings which is important for validation of simulation and the understanding of the experiments. 


\title{
Chapter 2
}

\section{Purpose and Structure}

The purpose of this thesis is to study the defect formation and their effect on optical properties in GaN by single and molecular ion irradiation and get insight into some experimental findings. For this purpose, simulation of ion irradiation is done by MD simulation code PARCAS [21-23].

This thesis consists of a brief summary part and five original publications, which are published in international peer-reviewed journals, presented with the permission of their publishers. The publications are referred to using boldface Roman numerals within the text.

The content is structured as follows. In this section, each publication included in this thesis are summarized. The author's own contribution is described as well as those of others. In chapter 3, structure, growth, applications and properties of $\mathrm{GaN}$ is briefly described. In chapter 4, several aspects of ion irradiation are described. The modelling methodology and analysis techniques are presented in chapter 5 and this is followed by the summarized results in chapter 6 . Finally, the closing remarks are provided in chapter 7 .

\subsection{Summary of original publications}

\author{
Publication I: Molecular dynamics simulations of damage buildup by atomic and molecular \\ projectiles in GaN
}

M. W. Ullah, A. Kuronen, F. Djurabekova, K. Nordlund and P. Karaseov, Proceedings of the 2011 Ion-Solid Interactions conference (Moscow aviation institute publisher, Moscow, Russia 250, (2011) 
This paper contains a preliminary study of irradiation induced damage in GaN. We used MD simulations to compare atomic and molecular ion irradiation of $\mathrm{GaN}$. The results showed indication of non-linear cluster effect in damage formation but there was large statistical variation in the data.

Publication II: Atomistic simulation of damage production by atomic and molecular irradiation in $\mathbf{G a N}$

M. W. Ullah, A. Kuronen, K. Nordlund, F. Djurabekova, P. A. Karaseov and A. I. Titov, Journal of Applied Physics 112, 043517 (2012)

This paper presents a comparative study of defect production in $\mathrm{GaN}$ by single $(\mathrm{P}, \mathrm{F}, \mathrm{Ar}$, $\mathrm{Ag})$ and molecular ion $\left(\mathrm{PF}_{4}, \mathrm{PF}_{2}\right)$ irradiation having energy of $0.05 \mathrm{keV} / \mathrm{amu}$. Although the same energy per mass was used, defect production was non-linear at the beginning of irradiation based on molecule size and mass. On the contrary, very strong defect recombination has been observed which lead to linearity in the final defect numbers.

\section{Publication III: Defect clustering in irradiation of GaN by single and molecular ions}

M. W. Ullah, A. Kuronen, F. Djurabekova, K. Nordlund, A. I. Titov and P. A. Karaseov Vacuum 105, $88(2014)$

Here we irradiated $\mathrm{GaN}$ by a single and a molecular ion with an energy of $0.6 \mathrm{keV} / \mathrm{amu}$ and reported the preliminary results. The measured photoluminescence (PL) decay time was found to be decreasing faster when irradiation was done by molecular ion compared to light ion irradiation. From irradiation simulations, we suggested that large defect clusters produced by molecular ion were responsible for the PL quenching. At the same time, more simulations were suggested to get better statistics.

\section{Publication IV: Effects of defect clustering on optical properties of GaN by single and molecular} ion irradiation 
M. W. Ullah, A. Kuronen, K. Nordlund, F. Djurabekova, P. A. Karaseov, K. V. Karabeshkin and A. I. Titov, Journal of Applied Physics 114, 183511 (2013)

As a further extension of previous study, more irradiation simulations were done and $\mathrm{Ag}$ was used as a projectile due to a comparable mass with $\mathrm{PF}_{4}$. In this publication, with large number of statistical data, we confirmed the findings of the previous study (Publication III) and showed that $\mathrm{Ag}$ is even more efficient than $\mathrm{PF}_{4}$ for defect cluster formation. Defect formation was explained by overlapping collision cascades and cascade density.

\section{Publication V: Atomistic simulation of Er irradiation induced defects in GaN nanowires} M. W. Ullah, A. Kuronen, A. Stukowski, F. Djurabekova and K. Nordlund, Journal of Applied Physics $116,124313,(2014)$

In this paper, a GaN nanowire was irradiated by the rare earth ion Er. Irradiation induced strain was observed in both $c$ and $a$ lattice parameters. Defects were found to be mostly concentrated at the surface region of the nanowire, which might be the reason for the experimentally observed intense surface yellow luminescence band.

\subsection{Author's own contributions}

The author carried out all the simulations in publications I-V. Analysis of the results and writing of the manuscripts were mainly carried out by the author. The photoluminescence decay time study in Publications III and IV and related experimental results and discussions were done by Platon Karaseov. In Publication $\mathbf{V}$, the strain calculation was done by Antti Kuronen and a modified version of the Voronoi defect analysis method was developed by Alexander Stukowski.

\subsection{Other works}

The author has also participated in the following scientific publications, which, however, are not part of this $\mathrm{PhD}$ thesis.

\section{Silicon crystal morphologies during solidification refining from Al-Si melts} Mohammad W. Ullah and T. Carlberg, Journal of Crystal Growth 318, 212 (2011) 
Simulation of surface solidification in direct-chill 6xxx aluminium billets

M. Erdegren, M. W. Ullah and T. Carlberg, IOP Conference Series: Materials Science and Engineering 27, 012013 (2012) 


\section{Chapter 3}

\section{Gallium Nitride}

In this chapter, an overview of GaN, namely crystal structure, growth technologies, applications and properties that are relevant to this thesis are described.

\subsection{Crystal structure}

GaN can have two crystal structures, the hexagonal and the cubic phase. The thermodynamically stable or natural structure is wurtzite (hexagonal) and a metastable zincblende (cubic) structure can be formed under controlled growth conditions [24]. Besides these two common structures, under high pressure GaN experiences a phase transition to rocksalt structure [25]. We used wurtzite (WZ) structure of GaN in this thesis because of its stability and wide use in the industry.

\subsubsection{Wurtzite}

The wurtzite crystal structure is a member of the hexagonal crystal system. The structure is closely related to the structure of hexagonal diamond. In both, each $\mathrm{Ga}$ atom is surrounded by $4 \mathrm{~N}$ atoms at the corners of a tetrahedron and vice versa. The primitive unit cell of WZ GaN consists of four atoms. Figure 3.1, shows the structure of a unit cell of GaN. The occupied $(\beta)$ symbols at tetrahedral sites and open $(\gamma)$ symbols represent gallium and nitrogen atoms, respectively. Ga atoms can either be at heights $(3 / 8) c$ above $\left(\beta_{1}\right)$ or below $\left(\beta_{2}\right)$ the $\mathrm{N}$ site, depending on the crystal polarity.

The stacking sequence along the $\left[\begin{array}{llll}0 & 0 & 0 & 1\end{array}\right] c$-direction is $\mathrm{AaBbAaBb}$. The $\mathrm{WZ}$ structure is represented by $a$ in the basal plane and $c$ perpendicular to the basal plane. The internal parameter $u$ is the ratio of anion-cation bond length and $c$ lattice parameter. In an ideal $\mathrm{WZ}$ structure, the values of axial ratio 


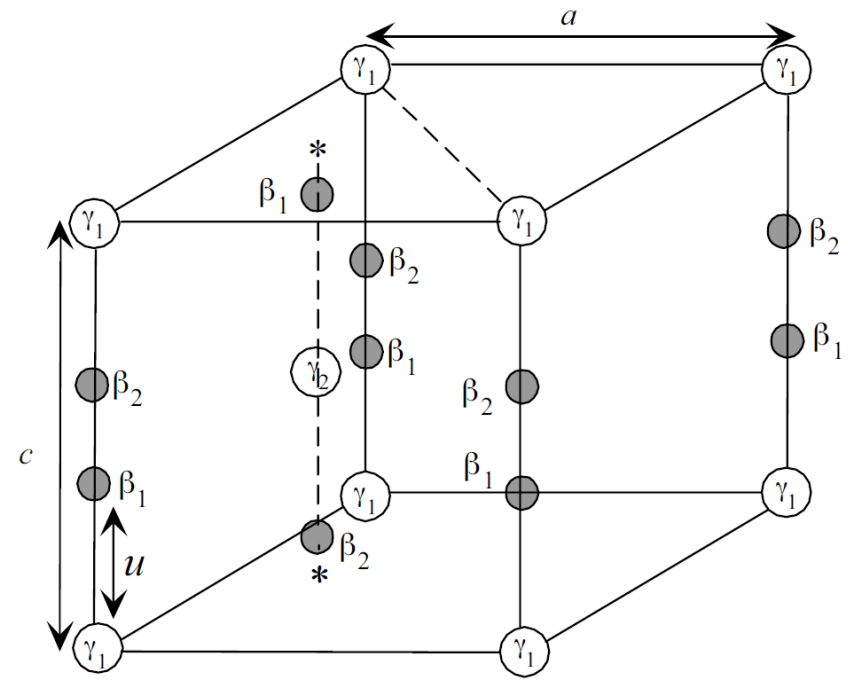

Figure 3.1: Schematic diagram of the GaN unit cell. Gray $\left(\beta_{1}, \beta_{2}\right)$ and open $\left(\gamma_{1}, \gamma_{2}\right)$ symbols represent occupancy sites for $\mathrm{Ga}$ and $\mathrm{N}$, respectively. $\beta$ sites are tetrahedral positions and only one family of $\beta$ sites can be simultaneously occupied by $\mathrm{Ga}$ atoms [26].

and internal parameter are $c / a=\sqrt{8 / 3}=1.633$ and $u=3 / 8=0.375$, respectively. In GaN, the $c / a$ ratio and the internal parameter $u$ slightly deviate from ideal values. Experimentally observed $c / a=1.634$ and $u=0.377$ for $\mathrm{GaN}$ [27]. Due to this deviation nearest neighbour distances also differ slightly: one with distance $c u$ and other three of length $\sqrt{1 / 3 a^{2}+(1 / 2-u)^{2} c^{2}}$ [28, 29]. The WZ primitive lattice vectors are

$$
\begin{array}{r}
\vec{a}=a(1 / 2, \sqrt{3 / 2}, 0) \\
\vec{b}=a(1 / 2,-\sqrt{3 / 2}, 0) \\
\vec{c}=a(0,0, c / a)
\end{array}
$$

The basis atoms are positioned at $(0,0,0),(0,0, u c), a(1 / 2, \sqrt{3} / 6, c / 2 a)$ and $a(1 / 2, \sqrt{3} / 6,[u+1 / 2] c / a)$. There are three planes in WZ structure of special importance, which are $\left(\begin{array}{llll}0 & 0 & 0 & 1\end{array}\right) c-,\left(\begin{array}{llll}1 & 1 & \overline{2} & 0\end{array}\right) a$ - and (1 $\left.\begin{array}{lll}1 & 0 & 0\end{array}\right)$ m-planes (Figure 3.2.

\subsection{GaN bulk crystal growth}

Around 1970, researchers (Pankove, Maruska and others) in Radio Corporation of America (RCA) laboratories pioneered the growth and characterization of $\mathrm{GaN}$ [31-33]. The currently available devices use GaN deposited by epitaxy on different substrates mainly on sapphire, $\mathrm{SiC}, \mathrm{GaAs}, \mathrm{Si}, \mathrm{LiGaO}_{2}$ and AIN [34]. Epitaxial methods like hydride vapor phase epitaxy (HVPE) [31, 35, 36], molecular 


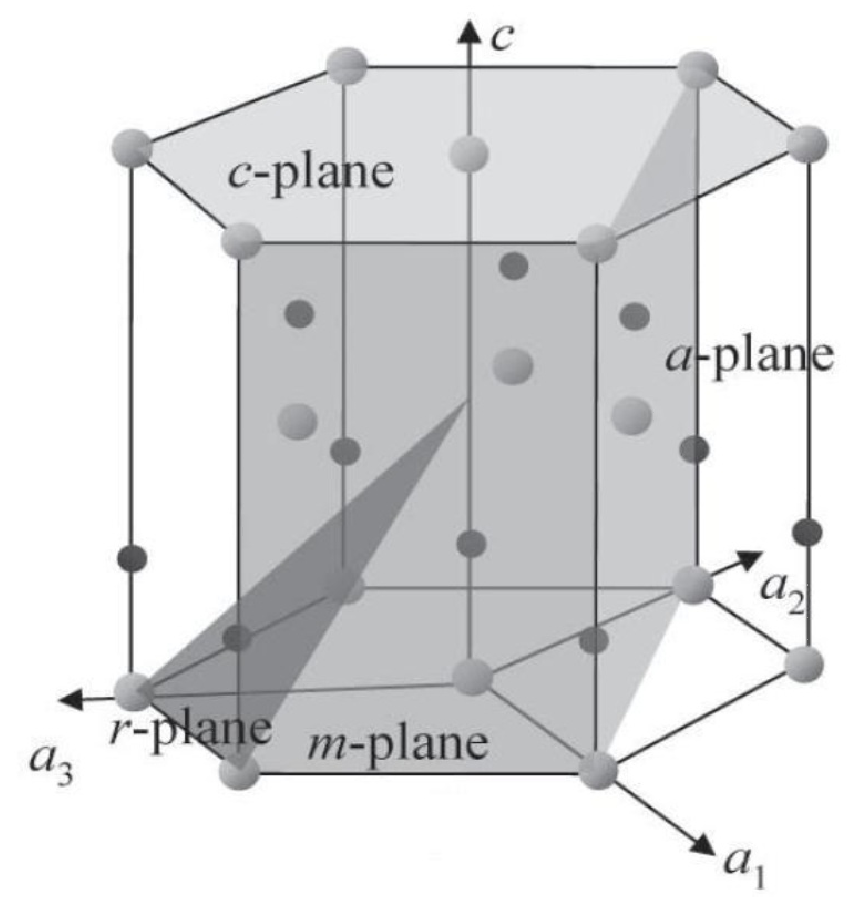

Figure 3.2: Important planes of wurtzite $\mathrm{GaN}$ [30].

beam epitaxy (MBE) [37, 38] and metalorganic chemical vapor deposition (MOCVD) [37, 39] are used for obtaining high quality GaN films. The resulting thin films exhibit high dislocation densities, high device leakage currents, reduced thermal conductivity, crack formation, short minority carrier lifetimes etc due to lattice constant ( $a$ and $c$ ) mismatch, coefficient of thermal expansion mismatch, different chemical composition than the epitaxial film etc. Due to the high melting temperature $(\sim$ $\left.2500^{\circ} \mathrm{C}[40,41]\right)$ and the high nitrogen pressure required for congruent melting $\left(\sim 6 \mathrm{GPa}\right.$ at $\sim 2220^{\circ} \mathrm{C}$ [42, 43]), it is difficult to grow single crystalline GaN. The standard techniques (Bridgman, Czochralski, Verneuil) for crystal growth are not applicable for GaN.

Three main growth methods currently used for preparing bulk GaN crystal are:

1. High pressure nitrogen solution growth process

2. Ammonothermal method

3. Na flux growth technology

\subsubsection{High pressure nitrogen solution growth process}

The high pressure and high temperature crystal growth process was developed by Porowski, Grzegory and co-workers [42, 44-47]. The experiments were performed in a high pressure chamber of an in- 
ternal diameter of $30 \mathrm{~mm}$ or $40 \mathrm{~mm}$ containing a furnace with an internal diameter of $14 \mathrm{~mm}$ (for temperatures up to $1500^{\circ} \mathrm{C}$ ) or $10 \mathrm{~mm}$ (for temperatures up to $1800^{\circ} \mathrm{C}$ ) with a boron nitride (BN) crucible partially filled with metallic gallium. Then oxygen was eliminated by annealing in vacuum. The crucible was then filled with pure nitrogen, compressed, and heated. The temperatures were stabilized with a precision of about $\pm 1^{\circ} \mathrm{C}$. The $\mathrm{N}_{2}$ pressure was kept at 0.8 to $1.7 \mathrm{GPa}$ at the temperature of $1300^{\circ} \mathrm{C}$ to $1700^{\circ} \mathrm{C}$ and the temperature gradient between the top and the bottom of the vessel was from 30 to $100{ }^{\circ} \mathrm{C} \mathrm{cm}^{-1}$ in 5-24 hour processes. In some recent works, it became possible to control the temperature with a precision of over $0.02{ }^{\circ} \mathrm{C}$ and the temperature gradient between 2 to $20^{\circ} \mathrm{C}$ $\mathrm{cm}^{-1}$. The GaN crystals were separated by a chemical treatment using boiling nitric acid [48].

The crystallization mechanism was driven by solid diffusion where a thin polycrystalline GaN film formed on the liquid Ga surface [46, 47]. Metallic gallium appeared to help the dissociation of nitrogen molecule into atomic nitrogen. This polycrystalline $\mathrm{GaN}$ is dissociated in the hotter part and transported to the cooler part of the solution and increases the nitrogen supersaturation which leads to the nucleation and growth of GaN single crystals.

\subsubsection{Ammonothermal method}

In the mid-1990s Dwiliński et al. had first demonstrated that it is possible to get crystalline GaN by a chemical reaction between gallium and ammonia, in the presence of alkali metal amides $\left(\mathrm{LiNH}_{2}\right.$ or $\mathrm{KNH}_{2}$ ) as mineralizers [49, 50]. GaN was synthesized using supercritical ammonia and metallic gallium, at the temperature of $500^{\circ} \mathrm{C}$ and the pressure in the range of 400 to $500 \mathrm{MPa}$. This process has been further improved by Kolis et al. where a GaN crystal was grown using ammonia as a solvent and GaN as a nutrient at $T=400^{\circ} \mathrm{C}$ and $P=240 \mathrm{MPa}$, using mineralizers sodium or potassium amide [51]. Very recently, this method has been further optimized using potassium azide $\left(\mathrm{KN}_{3}\right)$ as a mineralizer [52]. Platelets and prismatic needles shaped $\mathrm{GaN}$ crystals were produced at $500^{\circ} \mathrm{C}$ in 10 days, using $\mathrm{GaN}$ powder and/or crystals as a nutrient, the $\mathrm{KN}_{3}$ concentration being 1.3 or $1.6 \mathrm{~mol} \mathrm{l}^{-1}$.

At present, a tremendous progress has been made of this process especially to get larger size of the crystal. AMMONO, a Warsaw based company in collaboration with Nichia Corporation is commercially growing bulk GaN single crystal by the ammonothermal method [53]. They are producing up to 2-inch diameter high quality bulk $c$-plane $\mathrm{GaN}$ substrate and also non-polar $m$-plane, $a$-plane and semi-polar GaN wafers [54]. 


\subsubsection{Na flux growth technology}

At the end of 1990s Yamane et al. were first to report the growth of wurtzite GaN crystals up to 2 $\mathrm{mm}$ in diameter from the $\mathrm{Na}$ flux using sodium azide $\left(\mathrm{Na}_{3} \mathrm{~N}\right)$ as the Na source [55]. In this process, a sealed steel tube was used under high $\mathrm{N}_{2}$ atmosphere to introduce gallium and $\mathrm{Na}_{3} \mathrm{~N}$ at temperatures from 600 to $800^{\circ} \mathrm{C}$ and pressures less than $11 \mathrm{MPa}$ for a duration between 24 and 96 hours. A high $\mathrm{Na}$ content is required to form $\mathrm{GaN}$, but with increasing temperature less $\mathrm{Na}$ is required.

This technology was soon improved and optimized, and the growth of up to $3 \mathrm{~mm}$ large GaN single crystals was reported [56]. The experiments in the $\mathrm{BN}$ vessel with a conic-shaped inner cavity were performed at $750^{\circ} \mathrm{C}$ and $5 \mathrm{MPa}$ of $\mathrm{N}_{2}$ pressure for 200-300 hours growth time. Yields and morphology of $\mathrm{GaN}$ single crystals varied with the molar ratio $\mathrm{Na} /(\mathrm{Na}+\mathrm{Ga})$ in the starting composition. The crystal size was further increased to $5 \times 3 \mathrm{~mm}^{2}$ platelets and bulky prismatic crystals of $1 \mathrm{~mm}$ along the $c$-axis [57].

\subsection{Applications}

$\mathrm{GaN}$ and its alloys span a continuous range of direct band gap energies from visible to ultraviolet spectrum. This makes GaN attractive for optoelectronics device applications, such as light emitting diodes (LEDs), laser diodes (LDs) and ultraviolet detectors. GaN based green and blue LEDs in combination with GaAs based red LEDs form the basis of large full color displays and white light illumination. They are used in outdoor displays and signs, interior automotive lighting, traffic signals, etc. Shorter wavelength $(\sim 400 \mathrm{~nm})$ of GaN-based LDs, make is possible to store higher density of data on compact disks [58, 59].

$\mathrm{GaN}$ is also getting attention in the field of high power and high temperature electronics. This interest stems from two intrinsic properties of GaN. The first is wide band gap, which means that power devices can operate at high temperatures and there is less cost for structural design to minimize heat. The second property is the high breakdown field that with excellent electron transport properties makes GaN suitable for general electronics, microwave rectifiers, switches, high power and high frequency transistors etc [60]. GaN is a good candidate for space applications like radar and satellite communications due to inherent high radiation resistance. GaN based solid state power amplifiers are in development to replace currently used vacuum tube based traveling wave tube amplifiers in many space applications [61]. 


\subsection{Optical properties}

Gallium nitride $(\mathrm{GaN})$ is a direct band gap semiconductor having the room temperature band gap energy of $3.4 \mathrm{eV}$ [31]. GaN-based semiconductor lasers are ideal optical light sources for high density data storage applications. In a perfect semiconductor, electron-hole pairs will thermalize and accumulate at the conduction and valence band extrema, where they tend to recombine. In a direct band gap semiconductor with applied electric dipole transition, the probability of radiative electron-hole pair recombination is high. As a result, high quality direct band gap $\mathrm{GaN}$ is a strong emitter of band gap radiation.

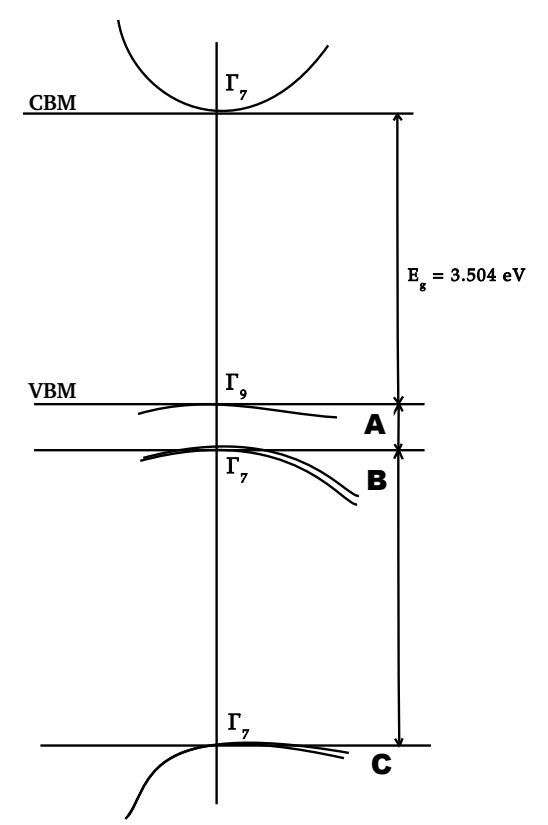

Figure 3.3: Band structure near the direct fundamental $\Gamma(k=0)$ gap of the wurtzite GaN. The three energy levels of the top valence bands are $\mathrm{A}\left(\Gamma_{9}\right), \mathrm{B}\left(\Gamma_{7}\right)$ and $\mathrm{C}\left(\Gamma_{7}\right)$ [62].

In $\mathrm{GaN}$, the top of the valence band and the bottom of the conduction band are at the same position in k-space $(k=0)$. The direct fundamental optical transition at or near this point between the upper valence band states and lowest conduction band minimum is most important for LEDs and lasers. The valence band is characterized by strongly coupled $\Gamma_{9}$, upper $\Gamma_{7}$ and lower $\Gamma_{7}$ levels, the holes in these three valence bands are called A-, B- and C-type holes or excitons, as shown in Fig. 3.3 [59]. Based on the above characteristics, Nakamura et al. first fabricated $p$ type GaN based high power blue LEDs [6, 63]. Colours from red to ultra-violet can be achieved through band gap tuning by combination of $\mathrm{GaN}$ with $\mathrm{In}(\mathrm{InGaN})$ or $\mathrm{Al}(\mathrm{AlGaN})$. Currently, commercially available blue ( $\sim 460$ $\mathrm{nm})$ and green $(\sim 520 \mathrm{~nm})$ LEDs are based on InGaN [8]. 
Extended and point defects can introduce states in the band gap which affect the optical properties. In low temperature PL spectra a peak at $3.41 \mathrm{eV}$ is observed, which is linked to the stacking faults. Another defect related broad band is observed at around $2.2 \mathrm{eV}$, the so called yellow luminescence (YL) band [64]. Implantation disorder has an effect on visible light absorption. As-implanted GaN undergoes color change from yellowish-brown to black depending on ion dose and implantation damage. Most of the PL and cathodoluminescence (CL) data from ion implanted GaN shows that defects act as effective non-radiative recombination centers, resulting in severe quenching of most characteristic luminescence peaks [11]. 


\section{Chapter 4}

\section{Ion Irradiation}

Ion irradiation is a process where energetic particles are bombarded into the surface of a solid substrate with enough energy (from electron-volt to mega-electron-volt energy range) to penetrate the surface region. The basic concepts of ion irradiation are slowing down of energetic ions, ion range, defect creation, sputtering, ion-beam mixing, the acceleration of kinetic processes and phase transformations.

\subsection{Stopping power and range}

When an energetic ion penetrates a solid, it undergoes a series of collisions with the atoms and electrons in the target. In these collisions, the energy loss of the incident particle depends on the energy, mass, and atomic number of the ion as well as on the mass, atomic number, and density of the substrate material. The energy-loss rate or stopping power of an energetic ion moving through a solid is determined by screened Coulomb interactions with the substrate atoms and electrons [65, 66]

$$
\lim \Delta E / \Delta x=d E / d x \equiv S(E)
$$

where $\Delta x \rightarrow 0, \Delta E=E_{0}-E_{1}$ is the energy loss per distance $\Delta x$ traversed and $d E / d x$ is called the stopping power (S) ( Fig. 4.1 (left) ). Typical units for the energy-loss rate are $\mathrm{eV} / \mathrm{nm}$ or $\mathrm{MeV} \mathrm{cm}{ }^{2}$ $\mathrm{mg}^{-1}$. There are three different mechanisms of energy loss:

1. Electronic stopping power: Inelastic collisions with the atoms in which the moving particle excites or ejects atomic electrons. 
2. Nuclear stopping power: Elastic collisions with the target nuclei in which energy is transmitted as translatory motion to a target-atom as a whole.

3. Radiative stopping power: Bremsstrahlung emission, Cherenkov radiation etc. These are important only at very high energies.
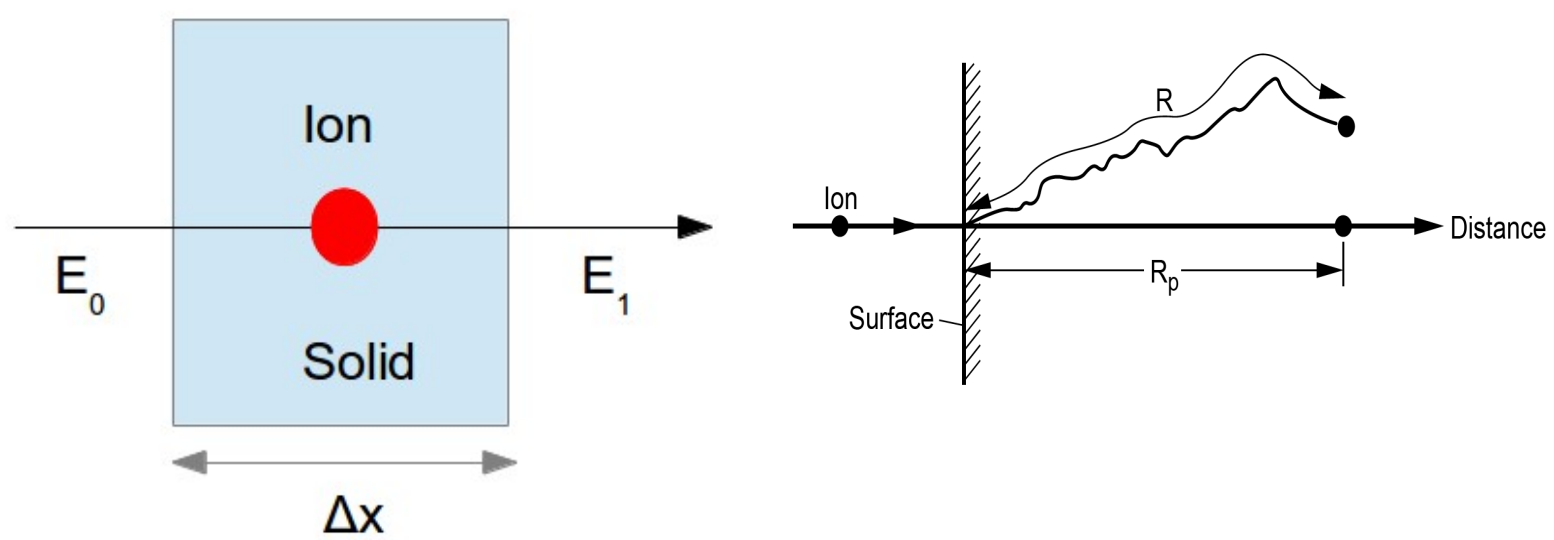

Figure 4.1: (Left) A particle (red) of incident energy $E_{0}$ moving through a solid of length $\Delta x$ having end energy $E_{1}$. (Right) An incident ion penetrates with a total path length $R$ and projected range $R_{P}$, along the direction parallel to that of the incident ion [65].

For most purposes, electronic and nuclear stopping powers are taken into consideration. The relative importance of the two stopping mechanisms depends on the energy $E$ and atomic number $Z$ of the particle: nuclear stopping predominates at low $E$ and high $Z$, whereas electronic stopping takes over at high $E$ and low $Z$ (Figure 4.2).

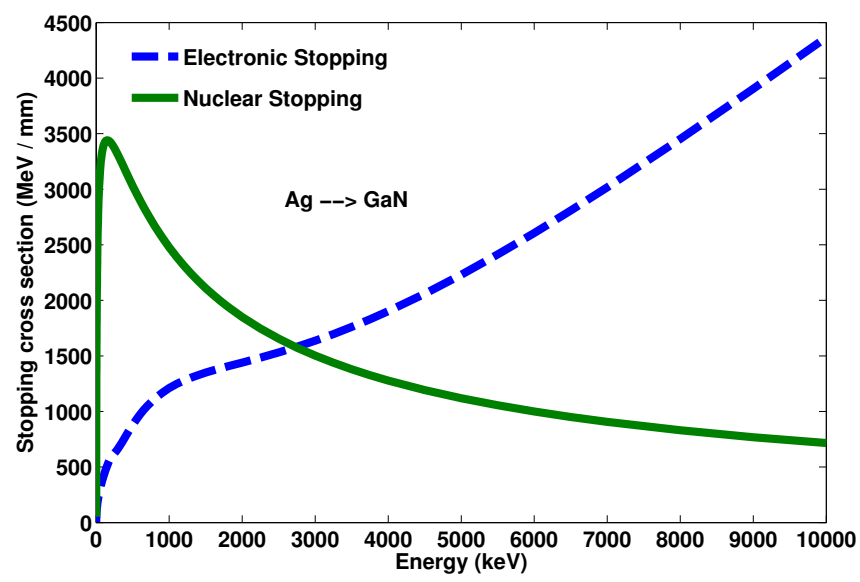

Figure 4.2: Electronic and nuclear stopping cross sections of $\mathrm{Ag}$ ions in $\mathrm{GaN}$ as calculated by SRIM2013 [67] computer code. 
Another important concept is the penetration depth or range, $R$ of the incident ion. The incident ion does not travel in a straight path due to collisions with target atoms. The actual integrated distance travelled by the ion is called the range, $R$. The ion's net penetration into the material, measured along the vector of the ion's incident trajectory, which is perpendicular to the surface, is called the projected range, $R_{P}$ ( Fig. 4.1-Right ). The distance travelled between collisions and the amount of energy lost per collision are random processes. So, all ions of a given type and incident energy do not have the same range. Instead there is a broad distribution in the depths to which individual ions penetrate. This distribution in ranges is called the range distribution and it's width the range straggling $\left(\Delta R_{P}\right)[65]$.

\subsection{Effects of ion irradiation on materials}

All kind of materials properties like mechanical, electrical, optical, magnetic and even superconductivity can be affected by ion irradiation. In general, effects can be divided into four main categories [68]:

1. Impurity introduction: imperfections can alter electrical and mechanical properties. Large number of impurity atoms may exert pressure on the neighbouring atoms which may lead to swelling in the material.

2. Atomic displacement: ion irradiation leads to displacement of atoms from their normal positions in the structure and forms vacancies and interstitials. Dissimilar atoms may interchange their positions and create antisites. If kinetic energy of the incoming particle is much higher, it can eject an atom from the target material to space, which is called sputtering.

3. Ionization: in this process an ion is created by removing or adding an electron from/to a neutral atom. At energies less than the ionization energy irradiation can cause the energy level of an electron to rise which is called excitation.

4. Energy release or thermal spikes: ion irradiation deposits energy to the atoms localized in a small volume in the target material. In organic materials, most of the deposited energy breaks chemical bonds and in metals, it creates heat. MD simulation of a $10-\mathrm{keV}$ event in $\beta-\mathrm{NiAl}$ shows that after tenths of ps, the maximum temperature exceeds $3000 \mathrm{~K}$ [69]. Depending on the energy density, the local temperature can rise above melting point which may form disordered or amorphous zone in the material.

Specific effects include: 
1. Mechanical properties: irradiation destroys the well defined lattice structure of crystalline materials, which changes the basic mechanical properties like hardness, ductility, toughness etc. Due to ion irradiation, hardness and strength increase when at the same time toughness decreases and a permanent strain is induced in the lattice. Radiation induced dislocations inhibit slip processes, which means, more energy is required to initiate slipping.

2. Electrical properties: ion irradiation has strong effect on the electrical conductivity of materials. Change of conductivity due to ion irradiation is different in metallic conductors, semiconductors and insulators. The introduction of point defects or irregularities into a metallic conductor increases electrical resistivity due to decreased mobility of charge carriers. In semiconductors and insulators, irradiation excites a large number of valence electrons to conduction band, creating electron-hole pairs. The excitation of valence electrons into the conduction band significantly increases the electrical conductivity for a short time. Ion irradiation induced defects also produce defect states in the band gap, which promotes easy access of electrons and holes.

3. Optical properties : by selecting proper ion energy and ion dose one can inject trace impurities that control luminescence, generate optical absorption bands or complex defect aggregates [70]. For instance, a broad and intense PL peak in the visible range $(\sim 680 \mathrm{~nm})$ and reduced transparency at high fluence has been observed by swift heavy ion irradiation on crystalline indium oxide thin film [71]. Also property like refractive index can be controlled efficiently by irradiation [72].

\subsubsection{Point and cluster defects}

Ion irradiation produces damage mainly in the form of point defects and defect clusters. When a particle passes through matter, the particle dissipates energy by exciting orbital electrons and by elastic collisions with the lattice atom. An elastic collision with sufficient energy can displace an atom from its normal lattice position. This displaced atom is called a primary knock-on atom (PKA) which, in turn, may displace other atoms, i.e., secondary knock-on atoms and so on, thus creating a cascade of atomic collisions, a collision cascade [65]. The displaced atom becomes an interstitial and the position the atom formerly occupied becomes a vacancy. Together they are referred to as a Frenkel pair. Point defects form along the tracks of the particle. At the end of the range, the atomic energy falls drastically (increase of $S(E)$ ) and collisions can not be treated as a two-body event, but rather as a many-body phenomenon. As a result, all the atoms in a small volume are set into motion and the local temperature increases, giving rise to defect clustering or a liquid like zone. In many cases, point defects are very mobile $(\mathrm{GaN}, \mathrm{Si})$ and many of them anneal out at room temperature, whereas extended defects like defect clusters are very stable. For instance in $\mathrm{Si}$, all simple point defects anneal 
out at temperature below $500{ }^{\circ} \mathrm{C}$, whereas cluster defects are particularly stable up to $800^{\circ} \mathrm{C}$ [73]. The probability of producing defect clusters increases with increasing PKA energy. In metals like $\mathrm{Cu}$, $\mathrm{Ag}$ and $\mathrm{Au}$, recoil energies above $25 \mathrm{keV}$ produce large defect clusters [74]. Evolution from point to cluster defects is attributed to several factors like high mobility of point defects, dense collision cascades, annealing of point defects, high-dose implantation and impurity concentration [73, 75].

\subsection{Ion irradiation of GaN}

In the fabrication of $\mathrm{GaN}$ based devices, ion irradiation is an attractive tool. It enables introducing selective area doping, dry-etching, electrical isolation, quantum well intermixing and so on. But all these processes are accompanied by defect production into the near-surface region, which plays an important role in device performance. Although extensive studies of $\mathrm{GaN}$ have been done for much of the past decades, knowledge on damage production in $\mathrm{GaN}$ under ion bombardment are still rather limited and far from being understood. Irradiation induced defects can have both positive and negative effects. For instance, reduction of the carrier lifetime is attributed to gallium vacancies, which has potential applications in ultrafast optical devices [11, 76].

The semiconductor saturable absorber mirror (SESAM) is an example of ultrafast optical device, successfully used in a wide range of solid state and fibre lasers. GaN-based SESAM with GaInN/GaN quantum wells has been demonstrated, which can be used to passively mode-lock blue lasers for producing ultra-short optical pulses [77,78]. The speed of this device is constrained by the lifetime of the photo-excited carriers, which can be as long as a few nanoseconds. In order to achieve sub-picosecond pulse duration, the absorption recovery time should attain ps levels. Ion irradiation induced defects in the form of traps and recombination centers can reduce the carrier decay time down to sub-picosecond levels. It has been reported that $\mathrm{Ne}^{+}$and $\mathrm{Ni}^{+}$irradiated InGaAs/GaAs QWs at room temperature can reduce carried decay time down to $0.54 \mathrm{ps}$ and $0.62 \mathrm{ps}$ (Figure: 4.3 ), respectively, and controlled creation of defects in GaInAs/GaAs quantum well heterostructures is possible for ultra-fast optoelectronic devices [79-81].

The works of Dhaka et al. [79, 80] indicated that the carrier decay time when irradiated with different kinds of projectiles depends on the total number of defects and their type. Previous studies have correlated the decay time with defect clusters due to the observation of defect clusters in transmission electron microscopy (TEM) [82]. Later on, by means of atomistic simulation, Björkas et al. [83] reported that increasing amount of large defect clusters decreases the carrier decay time and heavy ion irradiation can produce large defect clusters more efficiently than light ions. 


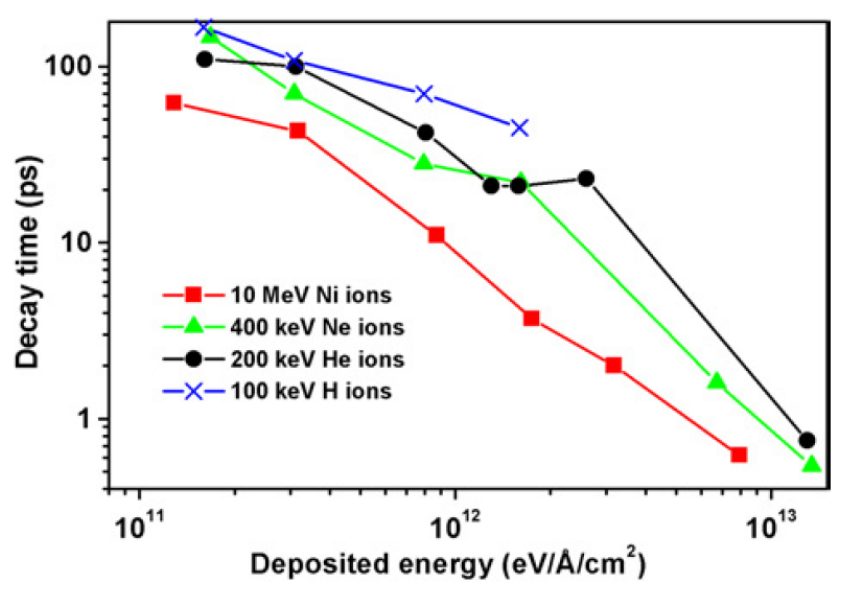

Figure 4.3: Carrier decay time as a function of deposited energy for different projectiles. The irradiation energy for each ion is chosen in such a way that similar penetration depth and deposited energy profiles are obtained. [80].

In $\mathrm{GaN}$, the damage buildup behaviour of atomic and molecular ions irradiation shows that molecular ions are more efficient in damage production than single ions in the near surface region, and this efficiency is attributed to the higher density of collision cascades produced by molecular ions [84]. The cascade density effect during ion irradiation was studied by maintaining the following parameter constant: ion energy normalized to ion mass, ion dose normalized to the number of displacements per atom (DPA) and ion beam flux normalized to DPAs ${ }^{-1}[14,85]$. Here, DPA $=\Phi \times g_{v} / n_{a t}$, where $\Phi$ is ion dose, $g_{v}$ is the SRIM [86] calculated number of ion-beam-generated lattice vacancies at the maximum of the nuclear energy loss profile, and $n_{a t}$ is the atomic density of the target.

Figure 4.4(a) illustrates that for the same DPA and energy per mass, a molecular ion $\left(\mathrm{PF}_{4}\right)$ produces more near-surface damage than single ions ( $\mathrm{P}$ and $\mathrm{F}$ ). Collision cascade density near the surface is higher for the molecular ion than the density of cascades of atomic ions (Fig. 4.4(b)). This is explained by the overlap of separate cascades composing the cluster of fluorine and phosphorus atoms in a narrow near-surface region to a depth of $\sim 10-12 \mathrm{~nm}$.

In summary, the work of Dhaka et al. and Björkas et al. demonstrate that ion irradiation induced defect clusters can speed up the operation of GaAs based semiconductor lasers by up to 2 orders of magnitude. In the case of GaN, the work of Kucheyev et al. shows a threshold like behaviour between the efficiency of damage buildup and the density of collision cascades where cluster ions produce denser cascades than single ions [14]. In the following, damage production in GaN by single and molecular ion irradiation has been studied by means of atomistic simulation based on the above contexts. 


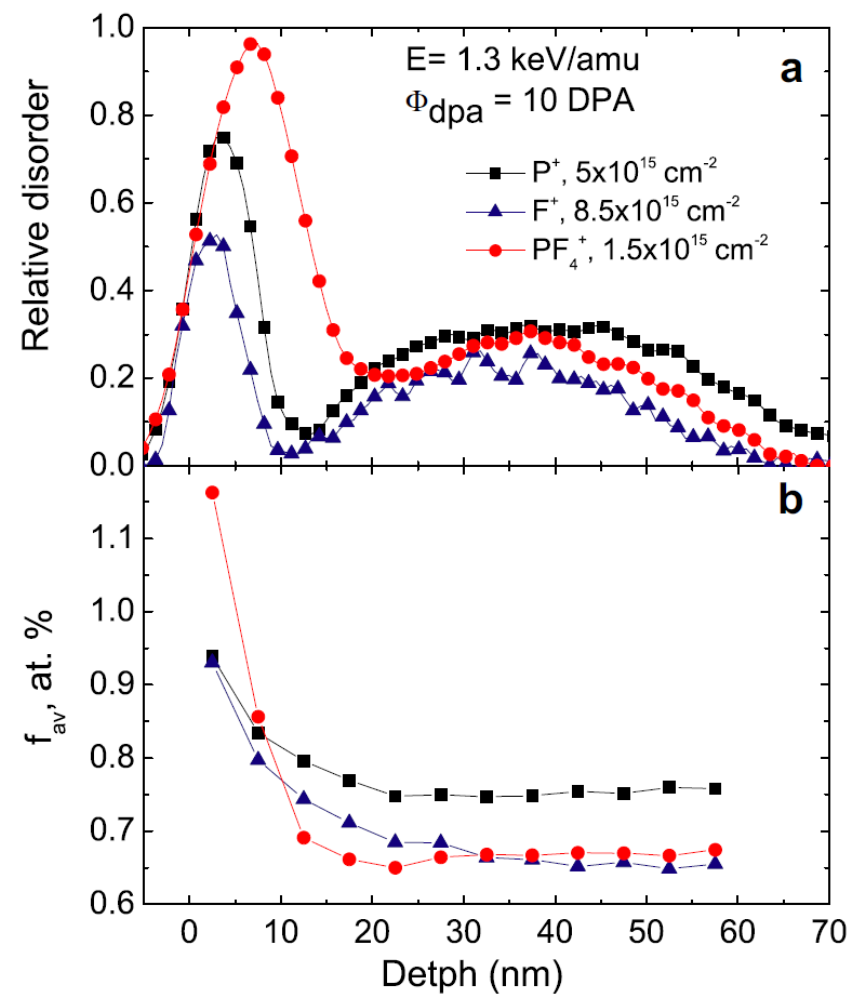

Figure 4.4: (a) Depth distribution of ion implantation induced disorder by $\mathrm{P}^{+}, \mathrm{F}^{+}$and $\mathrm{PF}_{4}^{+}$ions. (b) Depth distribution of the average density of collision cascades in $\mathrm{GaN}$ irradiated by the atomic and molecular ions listed in the legend in (a) with an energy of $1.3 \mathrm{keV} / \mathrm{amu}$. [87] 


\section{Chapter 5}

\section{Simulation Method}

In experiments, ion irradiation is done using an accelerator or ion implanter, and defects can be studied by Rutherford backscattering in combination with channeling (RBS/C), transmission electron microscopy (TEM) or X-ray diffraction (XRD). However, experimental studies can not always give insight of all processes, for instance the number of defects, cluster size, time evolution of defects can not be studied accurately. On the other hand, by computer simulation it is possible to have atomic scale understanding. How many defects are created, what is the initial state, and how these quantities depend on irradiation parameters can be studied by atomistic simulation.

In this section, the computer simulation method that has been used for this thesis is described. The main principles of MD is introduced and the interatomic potentials used for the simulations are explained.

\subsection{Molecular dynamics}

The basic idea of MD simulation is to calculate evolution of a system of particles in time by numerically solving classical equations of motion. In 1957, Alder and Wainwright had first used this method to calculate properties of many-body systems [88]. Figure 5.1], describes a simplified flowchart of MD algorithm.

The initial atomic coordinates $\left(\mathrm{r}_{i}\right)$ are given at the beginning of the simulation process. In most cases simulations are done at above $T=0 \mathrm{~K}$, so the initial temperature can be given by generating velocities from the Maxwell-Boltzmann distribution. Then the trajectories of atoms are evaluated by 


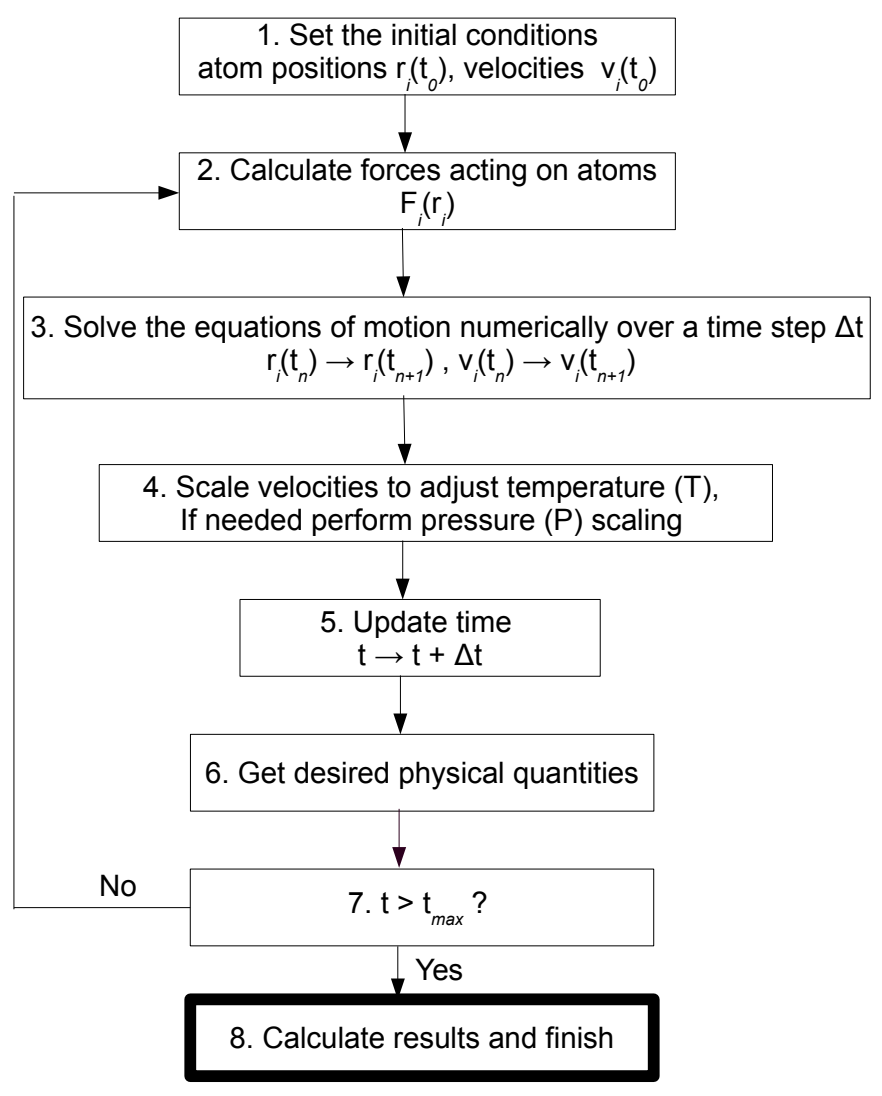

Figure 5.1: Basic steps in the molecular dynamics algorithm.

calculating all forces acting on each atom. The force acting on each atom is calculated from the gradient of potential energy $\mathrm{V}$.

$$
\boldsymbol{F}_{i}=-\nabla_{\boldsymbol{r}_{i}} V
$$

We can get acceleration $\left(\boldsymbol{a}_{i}\right)$ for atom $i$ having mass $m_{i}$ :

$$
\boldsymbol{a}_{i}=\frac{\boldsymbol{F}_{i}}{m_{i}}
$$

Then Newton's equations of motion are solved using a suitable integrator algorithm like Velocity Verlet [89] or Gear5 [90, 91]. In this work, MD simulation code PARCAS [21, 22] was used where the 
Gear5 predictor-corrector algorithm is employed. The choice of the time step $(\Delta t)$ is very important. It depends on the maximum velocity of the atoms in the system. The time step $\Delta t$ should be small enough that energy and momentum are conserved and large enough that the simulation does not become too slow. If $\Delta t$ is too large, the system will behave unphysically. In the case of irradiation simulation, an adaptive time step is used, which means that if there are energetic particles in the system, $\Delta t$ will decrease and with decreasing temperature $\Delta t$ will increase [23]. In the adaptive time step, $\Delta t$ is chosen as

$$
\Delta t_{n+1}=\min \left(\frac{\Delta x_{\max }}{v_{\max }}, \frac{\Delta E_{\max }}{F_{\max } v_{\max }}, c_{\Delta t} \Delta t_{n}, \Delta t_{\max }\right) .
$$

Here, $\Delta x_{\max }$ is the maximum allowed distance moved during any $\Delta t, \Delta E_{\max }$ is the maximum allowed change in energy, $v_{\max }$ and $F_{\max }$ are the highest speed and maximum force acting on any particle at $t$, respectively, $c_{\Delta t}$ prevents sudden large changes $\left(e . g . c_{\Delta t}=1.1\right)$, and $\Delta t_{\text {max }}$ is the time step for the equilibrated system.

To remove excess energy from the system deposited by an incoming ion or an energetic recoil or bring the initial system to some equilibrium temperature, the simulation cell is coupled with a heat bath. It is done by applying a thermostat algorithm, for instance, in this case Berendsen thermostat [92] is used to scale the atomic velocities by the factor

$$
\lambda_{B}=\sqrt{1+\frac{\Delta t}{\tau_{T}}\left(\frac{T_{0}}{T}-1\right)},
$$

where $\tau_{T}=\frac{1}{2 \gamma}(\gamma=$ damping constant $)$ is the time constant to determine the efficiency of the temperature control, $T_{0}$ is the desired temperature and $T$ is the initial temperature. $\tau_{T}$ should be chosen in such a way that system cools down to the desired temperature, but not so fast that it prevents natural temperature oscillations. In our bulk simulations, temperature control was activated all the time at the $x$ and $y$ periodic boundaries along lateral directions. In nanowire (NW) simulations the thermostat was activated at the bottom of the NW for 20 ps, after that the temperature of the entire cell was scaled to the desired value ( $300 \mathrm{~K})$ step by step. 
In the PARCAS code, it is also possible to perform pressure control. The pressure is scaled by changing the cell size. If the desired pressure is $P_{0}, P$ is the initial average pressure of the system and $\tau_{P}$ is the time constant, the scaling factor is

$$
\mu=\sqrt[3]{1-\frac{\beta \Delta t}{\tau_{P}}\left(P_{0}-P\right)}
$$

where $\beta=\frac{1}{B}$ ( $B=$ bulk modulus). In this study, pressure control was only used to relax the cell before irradiation to zero pressure to get the correct volume at the operating temperature.

Molecular dynamics becomes a very expensive computational method with increasing system size. So, to simulate a very large or bulk system an infinite lattice is approximated by using periodic boundary conditions $(\mathrm{PBC})$. It means that if an atom leaves the cell from one side $(+x / y / z)$, it will come back from other side $(-x / y / z)$ of the cell. Also, to prevent self interaction, simulation cell should be large enough [20]. In our simulations, the substrate was created by applying the PBC in all directions. In bulk irradiation, PBC was applied on $x-y$ directions and $z$ was open, whereas, no periodic boundary was used for the NW. The bottom two layers were fixed to prevent drifting during all simulations.

In PARCAS, the electronic stopping $\left(S_{e}\right)$, i.e., slowing down of the recoil while passing through the electrons is incorporated as a friction force for atoms with kinetic energies higher than $1 \mathrm{eV}$ and calculated using the SRIM version 2008.04 [86].

$$
\Delta v=\Delta t \frac{S_{e}}{m}
$$

where $\Delta t$ and $m$ are time step and ion mass, respectively. $\Delta v$ is subtracted from the recoil atom velocity.

\subsection{Interatomic potentials}

The interatomic potential describes the interaction between a pair of atoms or the interaction of an atom with a group of atoms. This is the heart of a MD simulation. The choice of the interatomic potential depends on the area of intended application, material type and computational time. Although MD potentials are classical, they are based on quantum mechanical principles. Classical MD takes Born-Oppenheimer approximation [93] into consideration, which states that electrons move so fast 
that they reach equilibrium before nuclei more significantly. Nuclei follow classical equations of motion due to their heavier mass than electrons.

\subsubsection{Analytical bond-order potentials}

The analytical bond-order potential (ABOP) of the Tersoff-Brenner [94-96] form is widely used and has been parametrized for materials, such as: $\mathrm{Si}, \mathrm{C}, \mathrm{Ge}, \mathrm{GaAs}, \mathrm{SiO}_{2}, \mathrm{SiC}$ etc. The potential formalism used for the GaN system in this thesis is of the ABOP type. The ABOP is based on Linus Pauling's bond order concept, which states that the strength of the interaction depends on the local environment. In particular, an atom with many neighbors forms weaker bonds than an atom with a few neighbors. The interaction also depends on the bond angle and the bond length.

The total potential energy in an ABOP of the Tersoff-Brenner form is written as a sum over individual bond energies:

$$
E=\sum_{i>j} f_{i j}^{c}\left(r_{i j}\right)[V_{i j}^{R}\left(r_{i j}\right)-\underbrace{\frac{b_{i j}+b_{j i}}{2}}_{\overline{b_{i j}}} V_{i j}^{A}\left(r_{i j}\right)] .
$$

Here $V_{i j}^{R}$ and $V_{i j}^{A}$ are the repulsive and attractive terms of the potential, respectively. The indices $i$ and $j$ run over the atoms of the system and $r_{i j}$ is the distance between atom $i$ and $j$. The term $f_{i j}^{c}$ is the cutoff function to limit the range of the potential.

The attractive and repulsive energies are pair potentials

$$
\begin{aligned}
V^{R}(r) & =\frac{D_{0}}{S-1} \exp \left(-\beta \sqrt{2 S}\left(r-r_{0}\right)\right) \\
V^{A}(r) & =\frac{S D_{0}}{S-1} \exp \left(-\beta \sqrt{2 S}\left(r-r_{0}\right)\right),
\end{aligned}
$$

where $D_{0}$ and $r_{0}$ are dimer bond energy and bond distance. If the binding energy $D_{0}$ and the groundstate frequency of the dimer molecule are known, then $\beta$ is simply given by

$$
\beta=k \frac{2 \pi c}{\sqrt{2 D_{0} / \mu}}
$$


where $k$ is the wave number and $\mu$ the reduced mass. The interaction is restricted to the nearest neighbours by a cut-off function, $f_{i j}^{c}\left(r_{i j}\right)$ :

$$
f^{c}(r)=\left\{\begin{array}{lc}
1, & r \leq R-D \\
\frac{1}{2}-\frac{1}{2} \sin \left(\frac{\pi}{2}(r-R) / D\right), & |R-r| \leq D \\
0, & r \geq R+D
\end{array}\right.
$$

where $R$ and $D$ are adjustable parameters, determining the cut-off range and interval. The bond order term $b_{i j}$, includes three-body interaction and angular dependencies,

$$
b_{i j}=\left(1+\chi_{i j}\right)^{-\frac{1}{2}}
$$

where

$$
\chi_{i j}=\sum_{k(\neq i, j)} f_{i k}^{c}\left(r_{i k}\right) g_{i k}\left(\theta_{i j k}\right) \exp \left(2 \mu_{i k}\left(r_{i j}-r_{i k}\right)\right) .
$$

Here, $\mu_{i k}$ is the fitting parameter, $\theta_{i j k}$ is the bond angle between bonds $i j$ and $i k$. The angular function $g_{i k}$ is given by

$$
g_{i k}\left(\theta_{i j k}\right)=\gamma\left(1+\frac{c^{2}}{d^{2}}-\frac{c^{2}}{d^{2}+\left(h+\cos \theta_{i j k}\right)^{2}}\right)
$$

where $\gamma, c, d$ and $h$ are adjustable parameters.

If $r_{b}$ is the equilibrium bonding distance and $E_{b}$ is the energy per individual bond, then $S$ can be determined from the Pauling relation

$$
E_{b}=-D_{0} \exp \left(-\beta \sqrt{2 S}\left(r_{b}-r_{0}\right)\right)
$$


The parameter sets for $\mathrm{Ga}-\mathrm{Ga}, \mathrm{N}-\mathrm{N}$ and $\mathrm{Ga}-\mathrm{N}$ were adjusted independently. Properties that are affected by other parameters were not included in the fitting procedure. Different dimer properties, structures of pure elements and compound including metastable phases can be described quite accurately by this potential. This gives a reasonable melting behaviour and solubility of nitrogen. The potential describes a wide range of $\mathrm{GaN}$ materials properties without including long-range interactions [97].

\subsubsection{Energetic short-range interaction}

The short range interactions between atoms are added to the potential after fitting the equilibrium properties correctly. This part of the potential is needed for high energy recoils where repulsive forces become stronger between two nuclei, such as during the simulation of ion irradiation. A common practice is to smoothly join the universal Ziegler-Biersack-Littmark (ZBL) [98] repulsive potential with the low energy part

$$
V_{Z B L}(r)=\frac{e^{2}}{4 \pi \varepsilon_{0}} \frac{Z_{1} Z_{2}}{r} \phi(r / a)
$$

where $Z_{1}$ and $Z_{2}$ are the charges of the nuclei, $r$ is the distance between nuclei, $e$ is the elementary charge and $a$ is a screening parameter

$$
a=\frac{0.8854 a_{0}}{Z_{1}^{0.23}+Z_{2}^{0.23}}
$$

where $a_{0}$ is the Bohr radius. The screening function $\phi(x)$ is given by

$$
\phi(x)=0.1818 e^{-3.2 x}+0.5099 e^{-0.9423 x}+0.2802 e^{-4028 x}+0.02817 e^{-0.2016 x} .
$$




\subsubsection{Lennard-Jones potential}

Apart from pure $\mathrm{GaN}$ interactions, we need the potential which can describe the interaction in $\mathrm{PF}_{4}$ and $\mathrm{PF}_{2}$ molecular ions. The cohesion of $\mathrm{PF}_{2}$ and $\mathrm{PF}_{4}$ molecules was modeled using the Lennard-Jones potential,

$$
V_{L J}(r)=4 \epsilon\left[(\sigma / r)^{12}-(\sigma / r)^{6}\right]
$$

It describes correctly bond lengths, bond energies and bond angles of the molecules (see Table 5.1. Figure 5.2). Dissociation energies $(\epsilon)$ were chosen as 4.6 and $1.6585 \mathrm{eV}$ [99-101] and the bond length parameters $(\sigma)$ were chosen as 1.408 and $2.131 \AA$ for the P-F and F-F bonds, respectively. The interactions at high energies were described by the Ziegler-Biersack-Littmark (ZBL) universal repulsive potential (see Section 5.2.2). ZBL potential was smoothly joined to Lennard-Jones potential by using the Fermi function to describe the interaction at high energies:

$$
f(r)=\frac{1}{1+\exp \left(r-r_{0}\right) / a},
$$

where $f(r)$ is the Fermi function. The total potential is

$$
V(r)=f(r) V_{Z B L}(r)+(1-f(r)) V_{L J}(r)
$$

\begin{tabular}{|c|c|c|c|}
\hline \multicolumn{4}{|c|}{ Geometrical parameters } \\
\hline Molecule & Parameters & Simulation & Reference \\
\hline \multirow{2}{*}{$\mathrm{PF}_{2}$} & $\ell$ & 1.579 & 1.579 \\
& $\theta$ & 98.83 & 98.5 \\
\hline \multirow{3}{*}{$\mathrm{PF}_{4}$} & $\ell_{1}$ & 1.54 & 1.54 \\
& $\ell_{2}$ & 1.59 & 1.61 \\
& $\theta_{1}$ & 105.09 & 103.7 \\
& $\theta_{2}$ & 166.41 & 164.6 \\
\hline
\end{tabular}

Table 5.1: Bond lengths $(\ell)$ and bond angles $(\theta)$ of $\mathrm{PF}_{2}$ and $\mathrm{PF}_{4}$ molecules (see Figure 5.2) used in the simulation and compared with experiment and theory [99-101]. Bond lengths are in $\AA$ and bond angles in degrees. 

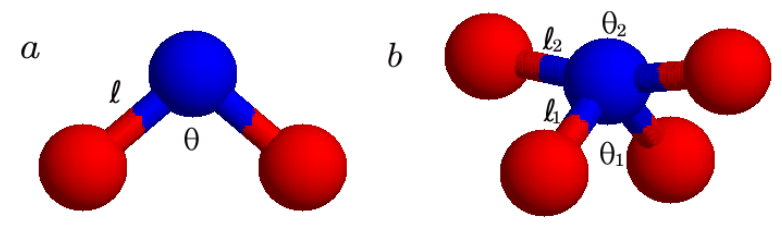

Figure 5.2: Geometries of (a) $\mathrm{PF}_{2}$ and (b) $\mathrm{PF}_{4}$ molecules

\subsection{Defect analysis}

The Voronoi cell method is used to identify vacancies, interstitials and antisites [21, 102]. Voronoi polyhedra were centered on each initial atom position and compared with the irradiated lattice. Polyhedra with no atoms were labeled vacancies, polyhedra with 2 or more atoms interstitials and polyhedra with one atom of the opposite type to the initial one were antisites.

In publication IV, a GaN nanowire was irradiated with rear-earth erbium (Er). In case of a free standing nanowire, the classical Voronoi cell method fails due to large elastic distortions. As a result, a large number of perfect crystal atoms are no longer confined to the Voronoi cell volume centered at their initial position, and a large number of unrealistic point defects is reported by the method. To be able to use the Voronoi cell method, we developed a preprocessing algorithm, which eliminates elastic displacements.

It is assumed that elastic displacement can be very large but relative elastic displacement change very slowly between two nearest neighbors. The algorithm starts with a fixed atom from the bottom of the irradiated nanowire and checks all the nearest neighbors within a certain cut off radius. The algorithm repositions each of the neighboring atoms to it's closest perfect lattice site. This process continues until all atoms in the crystal have been visited once and mapped onto ideal lattice sites. It is now possible to use the classical Voronoi method, since all atoms are located on ideal lattice sites. The process becomes a bit tricky when a nearest neighbor atom is close to more than one lattice site. In that case, the lattice site is chosen in such a way that it results in the smallest elastic displacement. The detailed explanation is given in Publication IV. In addition, cluster analysis was done by calculating the distance from each defect to all other defects. All defects that were within a fixed cut off radius from each other were interpreted to be a part of the same defect cluster.

The strain was calculated for each atom that was classified as not plastically displaced and had at least four of its nearest neighbors similarly classified. This classification was based on the preprocessing algorithm described above. The strain tensor was then calculated from the deformations of vertices of the neighbor atom tetrahedron [103]. 


\section{Chapter 6}

\section{Results}

\subsection{Comparative study of atomic and molecular projectiles}

The work presented in this section is based on publication I and publication II, where we studied the defect formation behaviour (total amount, type and position of the defects) in $\mathrm{GaN}$ for single and molecular ion projectiles. Of particular interest is, whether the damage increases non-linearly at the near-surface region for molecular projectiles as observed in experiment (Figure 6.1). Previously reported experimental data on implantation damage indicates very strong recovery of ion-generated defects in $\mathrm{GaN}$ [104]. Thus, we studied the time evolution of defects to have a better understanding about the dynamic annealing behaviour of $\mathrm{GaN}$.

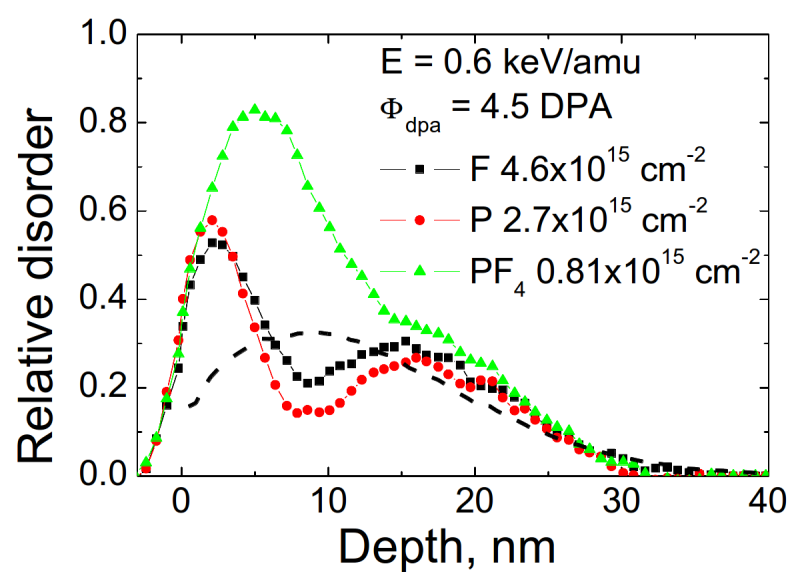

Figure 6.1: Depth profiles of relative disorder after implantation by different ions (as indicated in the legend) to doses resulting in 4.5 DPA.

The energies for $\mathrm{F}, \mathrm{P}, \mathrm{Ar}, \mathrm{Ag}, \mathrm{PF}_{2}$ and $\mathrm{PF}_{4}$ projectiles were $0.95,1.55,2,5.35,3.45$ and $5.35 \mathrm{keV}$, respectively. To compare between single and molecular ions, the energies were chosen in such a way that energy/atomic-mass was the same $(0.05 \mathrm{keV} / \mathrm{amu})$ for all atoms and molecules. The results 
show that there is a strong in-cascade damage recombination in GaN, it's even more pronounced for molecular ions than single ions (Figure 6.2-Left). The damage peak at about 0.25 ps is roughly 10 times higher for $\mathrm{PF}_{4}$ and 7 times higher for $\mathrm{PF}_{2}$ than the final damage after the cascade has cooled down. This recombination value $(\sim 10)$ is 2-3 times higher than in $\mathrm{Si}$ [21] and GaAs [105] but much less than in metals; for instance in Fe typical values are $~ 50$ [106]. There is a second peak in case of $\mathrm{PF}_{4}$ and $\mathrm{Ag}$ projectiles (Figure 6.2). This is attributed to the elastic wave caused by the collapse of big collision cascades produced by $\mathrm{PF}_{4}$ and $\mathrm{Ag}$. So, the second peak can be neglected.
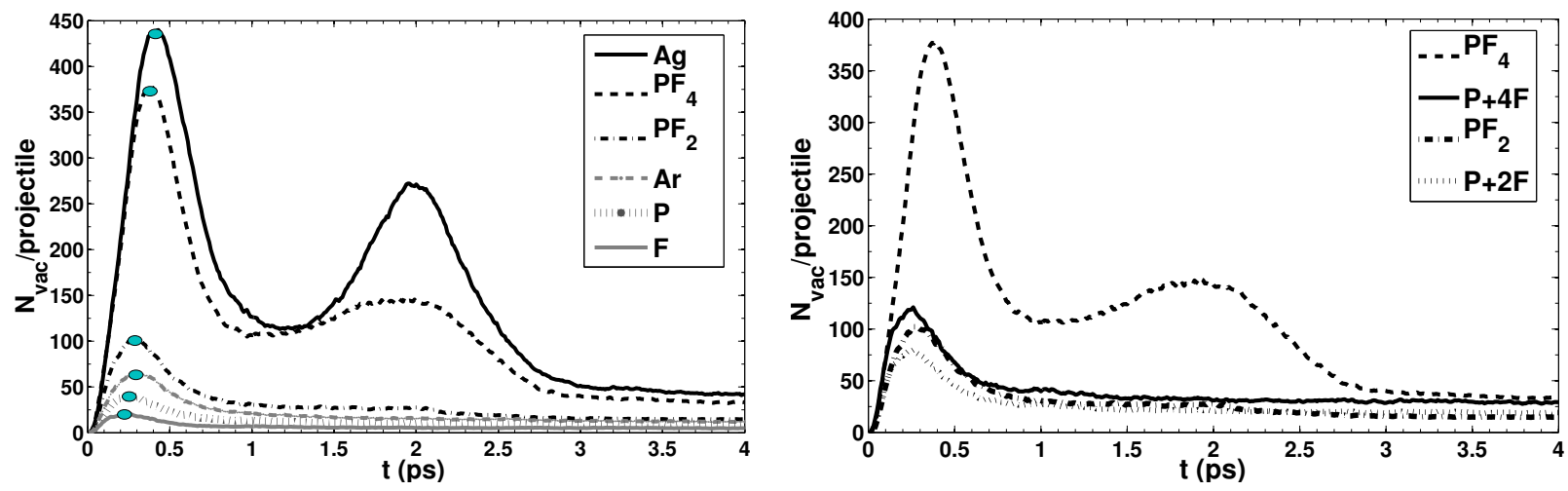

Figure 6.2: (Left) Time evolution of the number of defects for $\mathrm{F}, \mathrm{P}, \mathrm{Ar}, \mathrm{PF}_{2}, \mathrm{PF}_{4}$ and $\mathrm{Ag}$ projectiles. The ellipsoidal dots show the time step having the highest number of defects which were used in depth distributions in Figure 6.3. (Right) Time development of the number of defects of molecular projectiles compared with linear combinations of single projectiles $(\mathrm{P}+4 \times \mathrm{F}$ and $\mathrm{P}+2 \times \mathrm{F})$. From publication II.

Molecular effects in defect formation are characterized by plotting the number of vacancies calculated as linear combinations of $N_{\mathrm{vac}}(\mathrm{P})+2 N_{\mathrm{vac}}(\mathrm{F})$ and $N_{\mathrm{vac}}(\mathrm{P})+4 N_{\mathrm{vac}}(\mathrm{F})$ along with the number of vacancies produced by the molecular projectiles (Figure 6.2(right)). The idea is that if there are no molecular effects related to non-linearities in defect production, these curves should coincide with the corresponding molecular irradiation curves. For $\mathrm{PF}_{2}$, this is more or less the case. However, in the case of $\mathrm{PF}_{4}$ the amount of damage by the molecular projectile is 2-3 times higher than that of the linear combination of single ion damages. So, with increasing number of atoms in molecular projectile nonlinearity in instantaneous defects become more pronounced. However, after around 2.5 ps the curves coincide. Final number of defects shown in Table 6.1 also supports this linear behaviour.

The reason for the observed appearance of linearity in the case of molecular ions is the enhanced defect recombination property of GaN. Although a near-surface non-linearly disordered region has been observed experimentally at higher energies (Figure 4.4, 6.1), the total number of final defects for atomic and molecular ion irradiations does not exhibit a notable difference. 


\begin{tabular}{|c|c|c|c|c|c|}
\hline $\begin{array}{c}\text { Projectile } \\
\text { type }\end{array}$ & $\begin{array}{c}\text { Energy } \\
(\mathrm{keV})\end{array}$ & Vacancies & Interstitials & Antisites & Sputtered \\
\hline $\mathrm{F}$ & 0.95 & $5 \pm 1$ & $5 \pm 1$ & $4 \pm 1$ & 1 \\
\hline $\mathrm{P}$ & 1.55 & $9 \pm 2$ & $8 \pm 1$ & $8 \pm 1$ & 1 \\
\hline $\mathrm{PF}_{2}$ & 3.45 & $14 \pm 1$ & $16 \pm 1$ & $17 \pm 1$ & 4 \\
\hline $\mathrm{PF}_{4}$ & 5.35 & $29 \pm 3$ & $28 \pm 2$ & $32 \pm 3$ & 7 \\
\hline $\mathrm{Ag}$ & 5.35 & $37 \pm 5$ & $32 \pm 3$ & $33 \pm 3$ & 2 \\
\hline
\end{tabular}

Table 6.1: Average number of final defects and sputtered atoms produced by different projectiles after irradiation with a single ion. Uncertainties are the statistical errors of sets of 20 irradiations. From publication II.

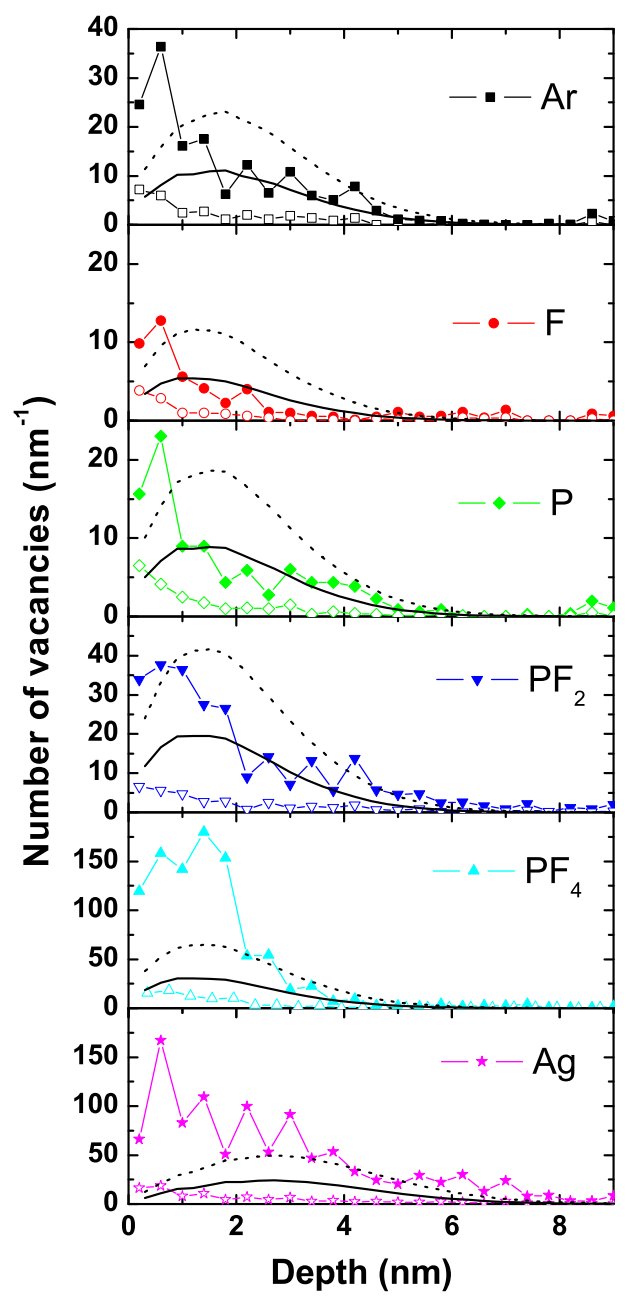

Figure 6.3: Depth distribution of instantaneous (closed symbols) and final (open symbols) vacancies produced in $\mathrm{GaN}$ by different projectiles. Also shown are the SRIM [86] predicted defect depth distributions using two different threshold displacement energies $E_{d}=25 \mathrm{eV}$ (solid line) and $12 \mathrm{eV}$ (dotted line). Closed symbol curves are calculated at the time of maximum defect number shown as ellipsoidal dots in Figure 6.2(Left).From publication II.

Figure 6.3 shows the defect (vacancy) depth distribution for all projectiles at the time step where the number of the defects is highest (no defect recombination), as well as the depth distribution of 
final defects. This figure also compares the results obtained by MD and SRIM which does not take into account in-cascade defect recombination. Neither the standard value of threshold displacement energy $\left(E_{\mathrm{d}}=25 \mathrm{eV}\right)$ nor even a twice lower value $\left(E_{\mathrm{d}}=12 \mathrm{eV}\right)$ can properly describe the MD defect generation. High defect density is apparent close to the surface and the defect peak for $\mathrm{PF}_{4}$ is four times higher than for $\mathrm{PF}_{2}$. This indicates non-linear damage production with increasing molecular size in near-surface region. Also, by comparing molecular ions $\left(\mathrm{PF}_{4}\right.$ and $\left.\mathrm{PF}_{2}\right)$ with single atomic ions (F, P and Ar) irradiation, we can see that former ions produce a significantly larger amount of lattice disorder close to the surface region.

\subsection{High energy irradiation and defect clustering}

It has been shown that in $\mathrm{GaN}$, molecular ions produce more structural damage in the surface proximity than atomic ions due to overlap of individual cascades (Fig 6.1). However, the question of what type of defects are produced in such cascades as well as their possible influence on photoluminescence (PL) properties of the material is still unclear. The collaboration from St. Petersburg State Polytechnic University (Russia) experimental group demonstrated that the PL decay time decreases faster for molecular projectiles than single ion projectiles when the energy per unit mass is the same for both $\mathrm{PF}_{4}$ and $\mathrm{P}$ projectiles (Fig 6.4). In the previous section, non-linear effects in the total number of final defects was analyzed. In this section, based on the above discussion, we will look at the defect types, particularly at defect clusters. For this study, we increased the energy to match with experiment for better comparison whereas in the previous section it was $\sim 10$ times lower than the experiment. This work is the topic of publications III and IV.

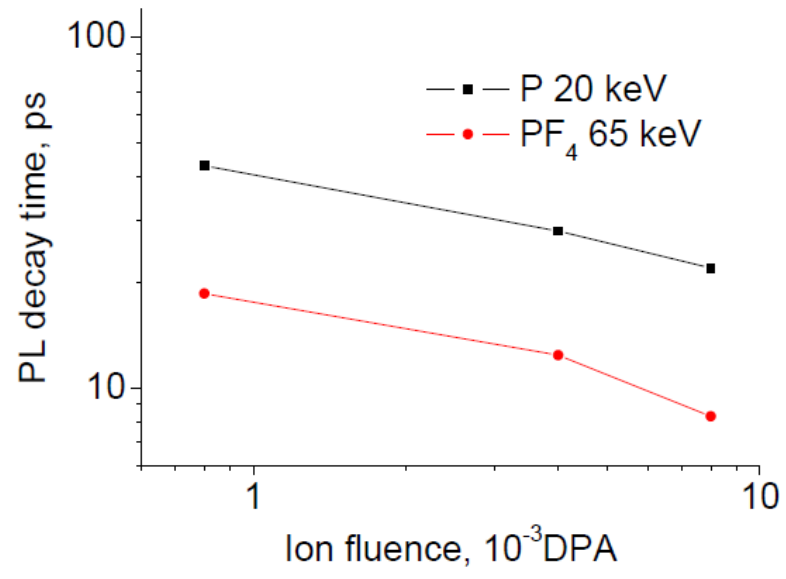

Figure 6.4: Photoluminecence decays times for $\mathrm{GaN}$ irradiated by single $(\mathrm{P})$ and molecular ion $\left(\mathrm{PF}_{4}\right)$ as a function of fluence expressed in units of displacement per atom (DPA) (conversion from fluence to DPA, see section 4.3), from publication IV. 


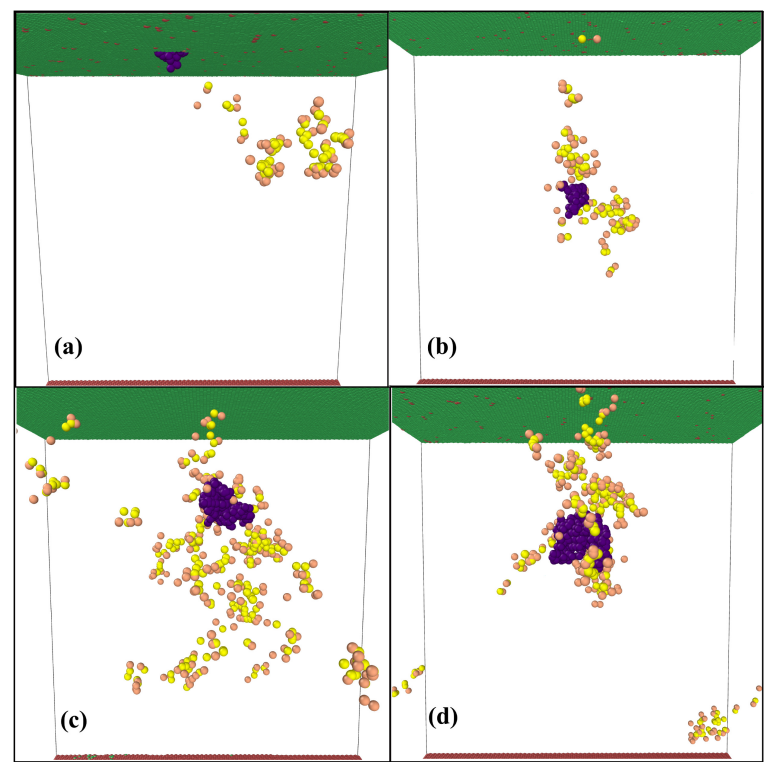

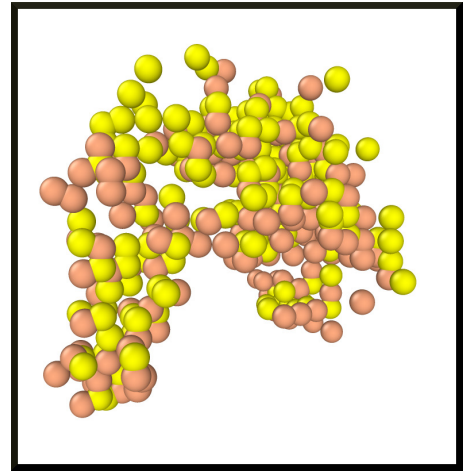

(b)

(a)

Figure 6.5: (a) Snapshots of defect cluster produced by F, $\mathrm{P}, \mathrm{PF}_{4}$ and Ag projectiles denoted by a, $\mathrm{b}, \mathrm{c}$ and d, respectively. The dark purple color shows the largest cluster. Light colors show the small clusters or point defects consisting of vacancies(yellow) and interstitials(orange). The projectile enters the substrate from the top. From publication IV. (b) Closer snapshot of the largest defect cluster produced by an Ag projectile.

Energies of 19, 11.4 and $65 \mathrm{keV}$ for $\mathrm{P}, \mathrm{F}$ and $\mathrm{PF}_{4}$ were used, respectively. Figure 6.5 (a) shows a visual comparison of defect clusters (vacancies and interstitials) produced by different projectiles. As we can see, the largest cluster produced by $\mathrm{PF}_{4}$ is much larger than the clusters produced by $\mathrm{F}$ and $\mathrm{P}$ irradiation. Defect distribution is wider and deeper in the case of molecular ions compared to the light ions.

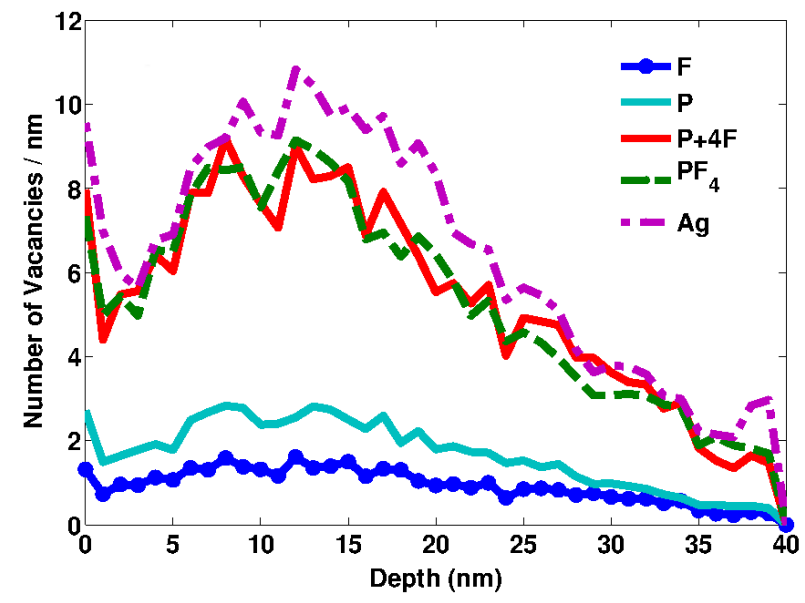

Figure 6.6: Vacancy depth distribution of different projectiles (as indicated in the legend). From publication IV. 
Figure 6.6 shows the damage-depth profiles for all projectiles and also the linear combination of $N_{\text {vac }}(\mathrm{P})+4 N_{\text {vac }}(\mathrm{F})$. Two peaks are observed in the curves: surface and near-surface region. The depth distributions of all point defects (Fig. 6.6) for $\mathrm{PF}_{4}$ and $\mathrm{P}+4 \times \mathrm{F}$ are almost overlapping. This clearly shows the linearity in total number of defects between single and molecular ion as observed in low energy irradiation as well (See Section 6.1).

The distributions of defect cluster sizes give a different picture compared with the total number of point defects described in the previous paragraph and Figure 6.6. The largest defect cluster produced by $\mathrm{PF}_{4}$ consists of more than 200 point defects whereas $\mathrm{P}+4 \times \mathrm{F}$ has produced defect cluster containing around 50 point defects at most (Figure 6.7). Table 6.2 gives the detailed data of Figure 6.7. The numbers of point defects and small clusters are almost the same for $\mathrm{P}+4 \times \mathrm{F}$ and $\mathrm{PF}_{4}$ but with increasing cluster size, the number of clusters obtained for $\mathrm{PF}_{4}$ starts to dominate. After cluster sizes 50-100, no clusters are visible for $\mathrm{P}+4 \times \mathrm{F}$.

\begin{tabular}{|c|c|c|c|c|c|}
\hline Type & \multirow{2}{*}{$\mathrm{P}$} & $\mathrm{F}$ & $\mathrm{P}+4 \times \mathrm{F}$ & $\mathrm{PF}_{4}$ & $\mathrm{Ag}$ \\
\cline { 1 - 5 } Size & & $28.0 \pm 0.6$ & $156 \pm 3$ & $146.0 \pm 1.3$ & $136 \pm 2$ \\
\hline 1 & $44.0 \pm 0.8$ & $11.4 \pm 0.3$ & $63.6 \pm 1.1$ & $58.4 \pm 0.6$ & $57 \pm 1$ \\
\hline $2-5$ & $18.0 \pm 0.4$ & $11.4 \pm 0.1$ & $11.5 \pm 0.3$ & $11.3 \pm 0.2$ & $13.0 \pm 0.2$ \\
\hline $5-10$ & $3.6 \pm 0.1$ & $2 \pm 0.1$ & $2.7 \pm 0.1$ & $4.2 \pm 0.1$ \\
\hline $10-20$ & $1.0 \pm 0.1$ & $0.40 \pm 0.03$ & $2.6 \pm 0.1$ & $0.80 \pm 0.04$ & $1.8 \pm 0.1$ \\
\hline $20-50$ & $0.30 \pm 0.03$ & $0.04 \pm 0.01$ & $0.40 \pm 0.04$ & 0.80 & $0.10 \pm 0.03$ \\
\hline $50-100$ & $0.01 \pm 0.01$ & $0.003 \pm 0.003$ & $0.02 \pm 0.01$ & $0.10 \pm 0.02$ & $0.40 \pm 0.01$ \\
\hline $100-200$ & $0 \pm 0$ & $0 \pm 0$ & $0 \pm 0$ & $0.03 \pm 0.01$ & $0.10 \pm 0.02$ \\
\hline $200-500$ & $0 \pm 0$ & $0 \pm 0$ & $0 \pm 0$ & $0.003 \pm 0.003$ & $0.03 \pm 0.01$ \\
\hline
\end{tabular}

Table 6.2: Size distribution of simulated defect production per ion for different projectiles and for the linear combination $\mathrm{P}+4 \times \mathrm{F}$. Data of this table is illustrated in Figure 6.7. From publication IV.

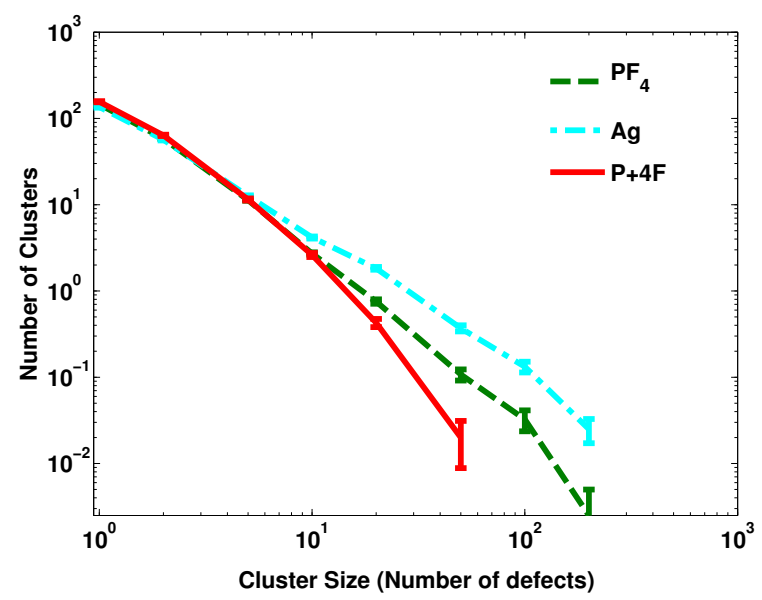

Figure 6.7: Cluster analysis of simulated defect production per ion for different projectiles (as indicated in the legend). Data is taken as an average of 400 simulations. Error bars show the standard error of the mean. From publication IV. 
Figure 6.4 shows quenching of the carrier decay time for molecular ions compared with single ions. It is known that carrier decay is correlated with defects. In literature, it was suggested that the change in the carrier decay time depends not only on the number of defects but also on the defect type. [80] In the case of group III-V semiconductors, ion irradiation in general shortens the carrier lifetime [76, 79, 80, 83]. Although GaN shows strong in-cascade damage recombination (see Section 6.1), the thermal stability of the large defect clusters created by molecular ions is high, up to $800^{\circ} \mathrm{C}$ [107]. Ion generated defects form different energy levels in the band gap. Most of these levels will be non-radiative recombination sinks. Hence, by ion irradiation we create a lot of new channels of non-radiative recombination. And even if luminescent transitions are still possible, the number of carriers recombining via these channels decreases [108]. Another explanation is interaction of ionbeam-generated defects with luminescent centers converting them to non-radiative [109]. Based on this, we suggest that one of the important mechanisms of faster PL decay time reduction after molecular ion irradiation is the formation of large and stable defect clusters.

\subsection{Mass effect on defect formation}

Beside single and molecular ions, we also used $\mathrm{Ag}$ which has the similar mass as $\mathrm{PF}_{4}$. In this case, the deposited energy during irradiation was equal for both projectiles. It has been reported previously that heavy ions are more efficient in reducing carrier decay time than light ions in semiconductor by producing defect clusters [83]. But, what about a molecular ion and a similar mass single ion? Which one is more efficient? From this point of view, we compared defect production between $\mathrm{Ag}$ (107.86 amu) and $\mathrm{PF}_{4}$ (106.93 amu). This work is based on publications II and IV.

The time evolution of defects at low energy irradiation (Figure 6.2 (left)) shows that the Ag curve is a little bit higher than the $\mathrm{PF}_{4}$ curve. The final number of defects is also slightly higher for $\mathrm{Ag}$ than for $\mathrm{PF}_{4}$ (Table 6.1).

From the snapshot of defects at a high energy (Figure 6.5), it is apparent that the largest defect cluster (dark purple color) is larger and defects are not as widespread for $\mathrm{Ag}$ than for $\mathrm{PF}_{4}$. In the case of the depth distribution of point defects (Figure 6.6), the $\mathrm{Ag}$ and $\mathrm{PF}_{4}$ curves are very close to each other. Thus, there is not much difference in the total number of final defects. On the other hand, if we take the same point defects and do the cluster analysis, we see a different scenario. Ag is producing a higher number of large defect clusters than $\mathrm{PF}_{4}$ (Figure 6.7). In the case of depth distribution of clusters containing more than 20 point defects, we see a clear higher peak for Ag also deeper in the sample than for all the other projectiles (Figure 6.8). 


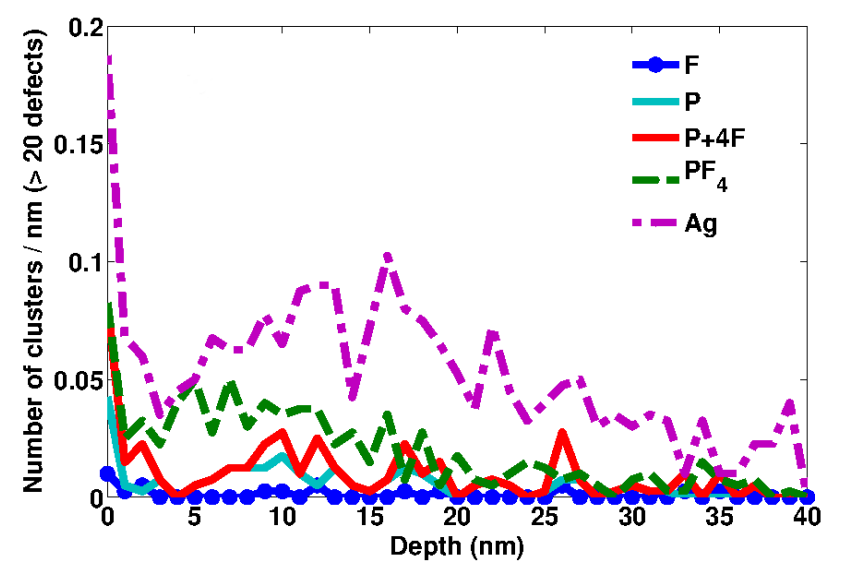

Figure 6.8: Depth distribution of clusters larger than 20 defects by different species. From publication IV.

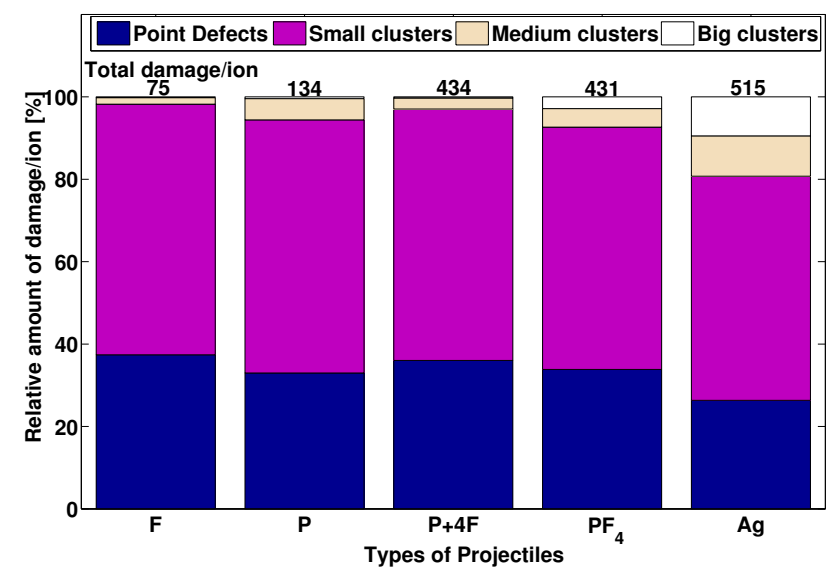

Figure 6.9: Total damage production per single or molecular ions and the percentage of different sized defect clusters. Small $=2-20$ defects, medium $=21-50$ defects, big $>50$ defects. From publication IV.

It is interesting to note that most of the defects are not point defects. In comparison with other projectiles, the percentage of point defects are the smallest for $\mathrm{Ag}(26 \%)$ and for $\mathrm{PF}_{4}$ it is $\sim 36 \%$. Small clusters dominate the distribution comprising up to $60 \%$ of the total number of defects for all the cases (Figure 6.9). Although the choice of cluster size limit is arbitrary, Ag and $\mathrm{PF}_{4}$ produced in total $19 \%$ and $7 \%$ of the defects as medium and large clusters, respectively. So, we clearly see that $\mathrm{Ag}$ is more efficient in large defect cluster production than $\mathrm{PF}_{4}$. This indicates that a heavy mass single ion may reduce the carrier decay time even faster than a molecular ion of similar mass.

These observations about light single, molecular and heavy mass ions can be explained based on the cascade density effect on defect formation [14]. Light ions ( $\mathrm{P}$ and F) produce very dilute collision cascades, so they mainly produce point defects and small clusters. $\mathrm{PF}_{4}$ breaks into individual atoms very close to the target surface (Figure 6.10), but collision cascades started by their atomic compon- 


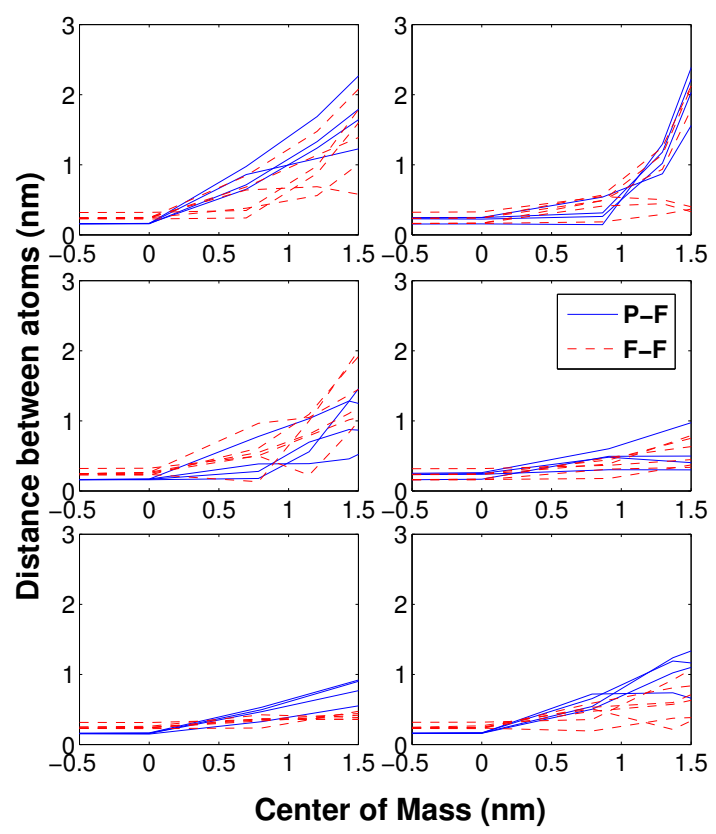

Figure 6.10: Dissociation of the $\mathrm{PF}_{4}$ molecule. Six different irradiation cases are shown. $x$-axis: center of mass of the molecule measured from the sample surface (zero of the axis), $y$-axis: interatomic distance between atoms. Atoms stay close to each other (interatomic distance less than 0.5-1 nm) until $\sim 1 \mathrm{~nm}$ depth. From publication II.

ents effectively overlap. This leads to a local increase of cascade density, which may fulfil the criteria for energy spike formation. As a result, the probability of formation of large stable defect cluster increases. Individual cascades become spatially separated deeper in the sample and the condition for large cluster formation vanishes. Although $\mathrm{Ag}$ and $\mathrm{PF}_{4}$ have comparable mass, $\mathrm{Ag}$ is more efficient in cluster production than $\mathrm{PF}_{4}$ due to it's concentrated mass. The nuclear energy loss rate is higher for heavy ions producing even denser collision cascade than $\mathrm{PF}_{4}$, which helps the formation of defect clusters or amorphous zones [11].

\subsection{Irradiation of a GaN nanowire}

In comparison with $\mathrm{GaN}$ based thin films or bulk, low dimensional structures like GaN nanowires (NW) are expected to offer potential advantages due to reduced dislocation density, less thermal expansion related lattice mismatch and high light extraction efficiency [110]. Like bulk GaN, ion irradiation used in GaN NW based devices also comes with the price of radiation induced damage [11, 111]. Lorenz et al. investigated defect related YL in RE implanted GaN NWs. They also reported irradiation induced lattice parameter expansion [112, 113]. Motivated by their work, we have irradiated GaN NW with Er to study the defect formation - especially the type and location of defects, 
which are not possible to pinpoint experimentally. Another focus of this study was to investigate the elastic strain induced by the irradiation defects. This section is based on publication $\mathbf{V}$.

Irradiation was done at the energy of $37.5 \mathrm{keV}$ on the $(0001)$ surface at $300 \mathrm{~K}$. The ion was placed above the simulation cell surface, and directed towards the surface at the incident angle of $20^{\circ}$ with respect to the NW axis. The NW contained 230000 atoms and had an average width of $10 \mathrm{~nm}$ and the length of $40 \mathrm{~nm}$.

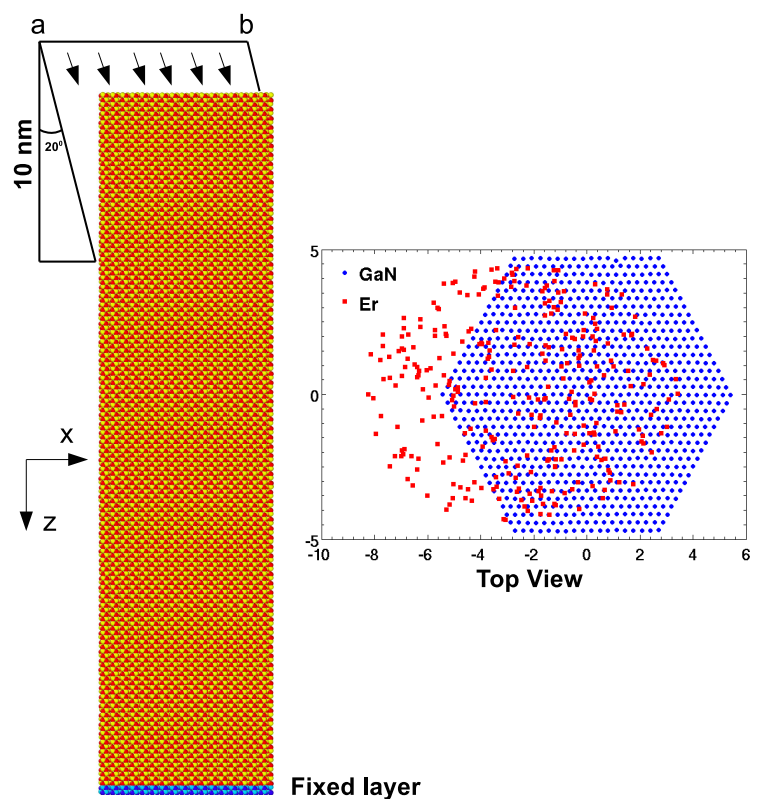

Figure 6.11: (Left) Schematic diagram of a simulation setup. The black lines and arrows show the estimated position and path of the projectiles, respectively. Projectiles from point a can hit the NW side down to $\sim 9 \mathrm{~nm}$ below the top and from point $\mathrm{b}$ they can reach the very end of the top surface. The blue bottom represents the fixed layer. (Right) Top view of the NW (blue circles) and random positions of the projectiles (red squares). From publication V.

Ten cumulative irradiations at random impact positions on the top surface of the NW were performed which corresponds to a fluence of $1 \times 10^{13} \mathrm{~cm}^{-2}$. For bulk irradiation, defect analysis was done by the classical Voronoi cell method but in the case of the nanowire due to large elastic strain a modified version of the Voronoi cell method was used (See Section 5.3). The local strain was calculated by using so called atomistic elasticity (AE) approach ([103], Chapter 5).

Figure 6.12 shows a quantitative analysis of the three diagonal strain components $\left(\epsilon_{x x}, \epsilon_{y y}, \epsilon_{z z}\right)$ of the strain tensor $\tilde{\epsilon}$. It shows how the local strain changes along the $z$ axis with increasing number of impacts. There are already large positive $\left(\epsilon_{z z}\right)$ and negative $\left(\epsilon_{x x}, \epsilon_{y y}\right)$ strain components after the first impact, which are caused by the longitudinal vibration of a free standing NW after the impact. There was no strain component at either end of the NW due to the fixed bottom layer and zero stress at the other end. All strain components are increasing at the irradiated end of the NW as a function 
of the number of impacts upto the depth of $z \sim 2-14 \mathrm{~nm}$, which agrees pretty well with the SRIM calculated ion range $11 \pm 4 \mathrm{~nm}$. This we attribute to the irradiation defects, as a high number of point defects is visible up to $z \sim 14 \mathrm{~nm}$ from depth distribution (Figure 6.13). Lorenz et al. have reported a similar observation of asymmetric broadening in the XRD $2 \theta-\omega$ curve of as-implanted GaN NWs and expansion of lattice parameter [112].

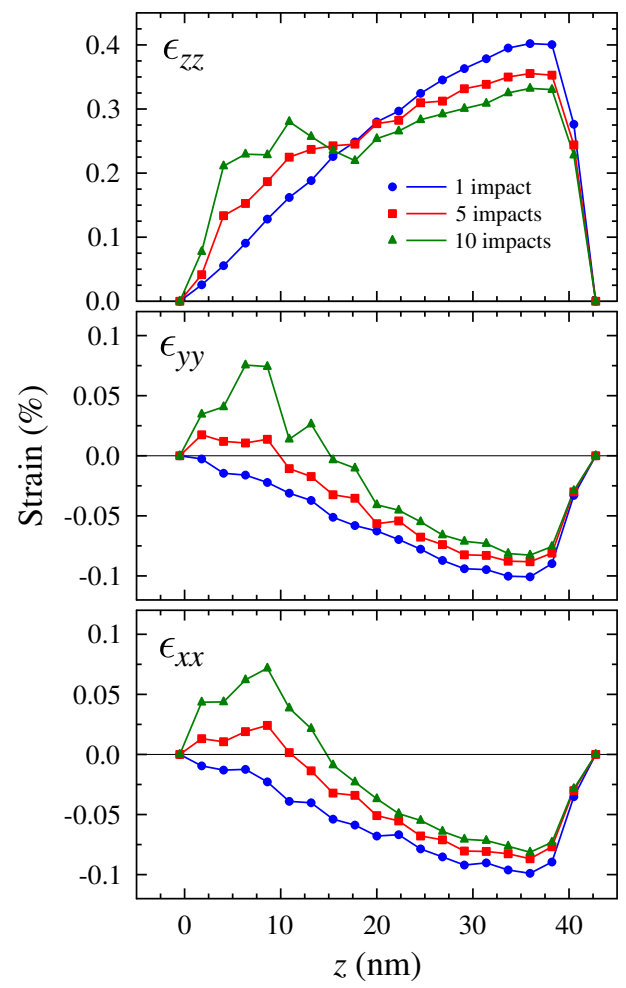

Figure 6.12: Three diagonal strain components as a function of NW major axis. The strain values are averaged over the NW cross section in the $x y$ plane and ten irradiation samples. The impact region is at $z=0-9 \mathrm{~nm}$ and the fixed bottom is at $z \sim 40 \mathrm{~nm}$. The strain components are plotted for the NW's after 1, 5 and 10 impacts. From publication V.

In experiments, strong YL band has been observed from the surface in both as-grown and RE implanted GaN NWs [114, 115] and they are linked with optically active surface defects [112, 116]. It is still not clear what kind of defects are responsible for the YL band and their position. Figure 6.14 reveals that defects are concentrated at a distance $\sim 4.5 \mathrm{~nm}$ outwards from the axis of the NW which is the position of outermost atomic layers of the NW. The same effect was observed in an MD simulation of an Ar implanted GaN NW where implantation was done perpendicular to the NW axis [117]. Besides point defects, large defect clusters ( 200 point defects) were also found and these can be stable even at high temperature. So, from high defect density at the surface, we can correlate the surface YL band with high density of surface defects. 


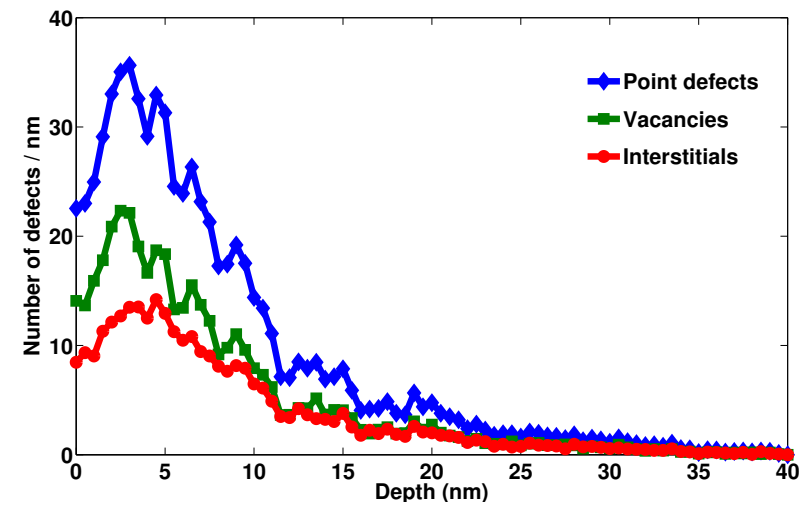

Figure 6.13: Depth distribution of point defects along the NW axis. The zero (0) point in $x$-axis represents the top surface as well as the impact region of the NW. Red circles, green squares and blue diamonds show interstitials, vacancies and combination of interstitials and vacancies, respectively. From publication $\mathbf{V}$.

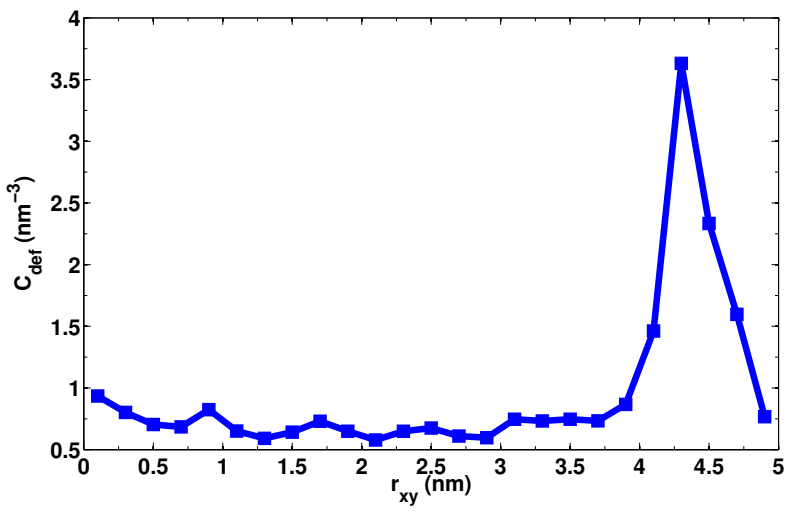

Figure 6.14: Radial density of defects as measured perpendicular from the NW axis. The smallest distance from the center to the surface of the $\mathrm{NW}$ is $\sim 4.5 \mathrm{~nm}$. From publication $\mathbf{V}$. 


\section{Chapter 7}

\section{Conclusions}

Detailed study of ion irradiation induced defects in $\mathrm{GaN}$ is not only important for fundamental knowledge about the formation of defect processes but also demanded by the growing semiconductor industry. This thesis is mostly motivated by experimental results, and the validity of theoretical results is established upon comparison with experiment. In this work, MD simulations were used to study the defect production in GaN by ion irradiation. The systems can be divided into two categories: the bulk and the nanoscale.

$\mathrm{GaN}$ was irradiated with single atomic ions such as $\mathrm{P}, \mathrm{F}, \mathrm{Ar}, \mathrm{Ag}$ and molecular ions such as $\mathrm{PF}_{2}$, $\mathrm{PF}_{4}$. For bulk simulations 0.05 and $0.6 \mathrm{keV}$ energy per mass was used for small (120000 atoms) and large (5.5 million atoms) systems, respectively. The main result of low energy irradiation can be divided into two parts. First, there was clear non-linearity in defect production between single and molecular ion irradiation when dynamic annealing was not taken into account. The instantaneous defect depth distribution of molecular ion $\left(\mathrm{PF}_{4}\right)$ is three times higher than linear combination of single ions $(\mathrm{P}+4 \times \mathrm{F})$.

Secondly, we saw a complete linearity of the total number of point defects at the end of the simulation when the sample cooled down. This behaviour of defect processes is indicative of a very strong defect recombination process in GaN even at room temperature. This result appears to be contradictory with experiment where non-linear defect formation has been observed. A likely explanation is the recombination of point defects on time scales longer than those accessible to molecular dynamics.

It was also observed experimentally that photoluminescence decay time was faster for molecular ion than single ion irradiation having the same energy per mass $(0.6 \mathrm{keV} / \mathrm{amu})$. We irradiated bulk $\mathrm{GaN}$ with the same energy as in experiments to investigate the effect of irradiation induced defects on the PL decay time. The main difference between single and molecular ion irradiation is the size 
distribution of defect clusters. Light ions mainly produce point defects and small clusters $(N<20$ defects), whereas molecular ions produce large defect clusters $(N>50$ defects). Thus, large and stable defect clusters can be one of the reasons for the faster PL decay time. Defects create different energy levels in the band gap. These levels act as non-radiative recombination sinks which leads to faster carrier decay.

Non-linearity in defect production is observed in the simulations when defect clusters were taken into account instead of individual point defects. Depth distribution of $\mathrm{PF}_{4}$ defect clusters produce a higher peak than $\mathrm{P}+4 \times \mathrm{F}$, which was in line with experiments. We also compared defect production between a molecular ion $\left(\mathrm{PF}_{4}\right)$ and a similar mass single ion $(\mathrm{Ag})$. Defect clusters produced by Ag were larger in size and also higher in number than $\mathrm{PF}_{4}$. From this result, we suggest that the heavy ions produce more disordered region than the similar mass molecular ions which may cause even faster decrease in PL decay time.

Due to the potential advantages and growing industrial interest, a defect production study was also done for a $\mathrm{GaN}$ nanowire by irradiating rare-earth ions (Er). Irradiation induced defects (point and cluster) change the lattice parameter of the NW. In agreement with experiment, the $c$-lattice parameter expands more than $a$. The large surface-to-volume ratio of the nanowire affects defect formation in such a way that they are mostly concentrated at the surface region, which might be the reason for the experimentally observed intense surface YL band. 


\section{Acknowledgements}

I would like to thank the former head of the Department of Physics at the University of Helsinki, Prof. Juhani Keinonen, the current head Prof. Hannu Koskinen, as well as Prof. Jyrki Räisänen, the head of the Division of Materials Physics, for providing the opportunity to conduct the research presented in this thesis. Financial support from Academy of Finland is greatly acknowledged.

Many thanks are due to Prof. Kai Nordlund, without whom I would not be here today. I am thankful to Kai for the positive attitude, good ideas and most importantly for having faith in me at first place. Whenever I had a discussion with Kai, the next article was almost done and same goes for the thesis as well.

I am forever grateful to my supervisor Doc. Antti Kuronen, without his support and encouragement, I could not have finished this thesis. You have guided me from the very first day with never ending patience. I am quite sure that I am the only student here, who has to discuss with his supervisor almost every day, even three or more times a day! You were always kind to me, answering with smile every question. I even knocked your door to talk about something completely irrelevant and stupid!

I extend my warmest thanks to my co-supervisor Doc. Flyura Djurabekova for all the discussions and new ideas. Thanks for her guidance, regarding both current and future work. This thesis is the result of fruitful collaboration with St.Petersburg State Polytechnic University. I especially thank Prof. Platon Karaseov for explaining me the experiments and add valuable experimental results in my thesis. I would also like to thank you for those inspiring pies!

In last four years my office mates have become true friends. I am grateful and lucky to have friends like Annika, Aleksi, Konstantin and Yi-Nan. Thank you Annika for being so lovely, caring, above all making work place fun, you are the best! Aleksi, Konstantin, thank you very much for being amazing friends and help me with all those work related stuffs that I could not solve! I warmly thank all other friends and co-workers in the accelerator lab, for creating a welcoming atmosphere where I spent most of the time during my thesis. 
Finally, I want to thank my parents for everything. You made me the man I am today. Thanks to my sister (Susama) and brother (Sumit) for being my best friends not just siblings. I love you guys! Thanks to all my friends who made their mark in my life.

Helsinki, 24th November 2014

Mohammad Wali Ullah 


\section{Bibliography}

1. W. C. Johnson, J. B. Parsons, and M. C. Crew, "Nitrogen compounds of gallium. III," J. Phys. Chem., vol. 36, p. 2651, 1932.

2. D. Ehrentraut, E. Meissner, and M. Bockowski, eds., Technology of Gallium Nitride Crystal Growth. Springer-Verlag Berlin Heidelberg, 2010.

3. H. Amano, N. Sawaki, I. Akasaki, and Y. Toyoda, "Metalorganic vapor phase epitaxial growth of a high quality GaN film using an AlN buffer layer," Appl. Phys. Lett., vol. 48, p. 353, 1986.

4. I. Akasaki, H. Amano, Y. koide, K. Hiramatsu, and N. Sawaki, "Effects of AlN buffer layer on crystallographic structure and on electrical and optical properties of $\mathrm{GaN}$ and $\mathrm{Ga}_{1-x} \mathrm{Al}_{x} \mathrm{~N}$ $(0<\mathrm{x} \leq 0.4)$ films grown on sapphire substrate by MOVPE," J. Cryst. Growth, vol. 98, p. 209, 1989.

5. H. Amano, M. Kito, K. Hiramatsu, and I. Akasaki, "P-type conduction in Mg-Doped GaN treated with low-energy electron beam irradiation(LEEBI)," Jpn. J. Appl. Phys., vol. 28, p. L2112, 1986.

6. S. Nakamura, T. Mukai, and M. Senoh, "Candela-class high-brightness InGaN/AlGaN doubleheterostructure blue-light-emitting diodes," Appl. Phys. Lett., vol. 64, p. 1687, 1994.

7. A. Y. Polyakov, "Radiation effects in GaN," in GaN and ZnO-based Materials and Devices (S. Pearton, ed.), p. 251, Springer-Verlag Berlin Heidelberg, 2012.

8. J. Y. Duboz, "GaN as seen by the industry," Phys. Stat. Sol. (a), vol. 176, p. 5, 1999.

9. M. S. Shur, R. Gaska, and A. Bykhovski, "GaN-based electronic devices," Solid-State Electronics, vol. 43, p. 1451, 1999.

10. S. Pearton, ed., GaN and ZnO-based Materials and Devices. Springer-Verlag Berlin Heidelberg, 2012.

11. S. O. Kucheyev, J. S. Williams, and S. J. Pearton, "Ion implantation into GaN," Materials Science and Engineering, vol. 33, p. 51, 2001.

12. A. I. Titov, S. O. Kucheyev, V. S. Belyakov, and A. Y. Azarov, "Damage buildup in Si under bombardment with MeV heavy atomic and molecular ions," J. Appl. Phys., vol. 90, no. 8, pp. 3867-3872, 2001. 
13. A. I. Titov, P. A. Karaseov, A. Y. Azarov, and S. O. Kucheyev, "Effects of the density of collision cascades: Separating contributions from dynamic annealing and energy spikes," Nucl. Instr. Meth. Phys. Res. B, vol. 267, no. 16, p. 2701, 2009.

14. S. O. Kucheyev, A. Y. Azarov, A. I. Titov, P. Karaseov, and T. M. Kuchumova, "Energy spike effects in ion-bombarded GaN,” J. Phys. D: Appl. Phys., vol. 42, p. 085309, 2009.

15. N. Parikh, A. Suvkhanov, M. Lioubtchenko, E. Carlson, M. Bremser, D. Bray, R. Davis, and J. Hunn, "Ion implantation of epitaxial GaN films: damage, doping and activation," Nucl. Instr. Meth. B, vol. 127, p. 463, 1997.

16. J. S. Williams, "Ion implantation of semiconductors," Mater. Sci. Eng. A, vol. 253, p. 8, 1998.

17. C. Ronning, E. P. Carlson, D. B. Thomson, and R. F. Davis, "Optical activation of Be implanted into GaN," Appl. Phys. Lett., vol. 73, p. 1622, 1998.

18. B. J. Pong, C. J. Pan, Y. C. Teng, G. C. Chi, W. H. Li, K. C. Lee, and C.H. Lee, "Structural defects and microstrain in GaN induced by Mg ion implantation," J. Appl. Phys, vol. 83, p. 5992, 1998.

19. A. R. Leach, Molecular Modelling: Principles and Applications. Harlow, England: Peasrson Education, second ed., 2001.

20. M. P. Allen and D. J. Tildesley, Computer Simulation of Liquids. Oxford, England: Oxford University Press, 1989.

21. K. Nordlund, M. Ghaly, R. S. Averback, M. Caturla, T. Diaz de la Rubia, and J. Tarus, "Defect production in collision cascades in elemental semiconductors and FCC metals," Phys. Rev. B, vol. 57, no. 13, pp. 7556-7570, 1998.

22. M. Ghaly, K. Nordlund, and R. S. Averback, "Molecular dynamics investigations of surface damage produced by keV self-bombardment of solids," Phil. Mag. A, vol. 79, no. 4, p. 795, 1999.

23. K. Nordlund, "Molecular dynamics simulation of ion ranges in the $1-100 \mathrm{keV}$ energy range," Comput. Mater. Sci., vol. 3, p. 448, 1995.

24. T. Hanada, "Basic Properties of $\mathrm{ZnO}, \mathrm{GaN}$, and Related Materials," in Oxide and Nitride Semiconductors (T. Yao and S. Hong, eds.), pp. 1-19, Springer Berlin Heidelberg, 2009.

25. I. Vurgaftman and J. R. Meyer, "Band parameters for nitrogen-containing semiconductors," J. Appl. Phys., vol. 94, p. 3675, 2003.

26. P. Ruterana, A. M. Sánchez, and G. Nouet, "Extended defects in wurtzite GaN layers: Atomic structure, formation, and interaction mechanisms," in Nitride Semiconductors (Handbook on Materials and Devices) (P. Ruterana, M. Albrecht, and J. Neugebauer, eds.), pp. 379-438, Wiley-VCH Verlag GmbH, Weinheim, Germany, 2003. 
27. O. Ambacher, J. Majewski, C. Miskys, A. Link, M. Hermann, M. Eickhoff, M. Stutzmann, F. Bernardini, V. Fiorentini, V. Tilak, B. Schaff, and L. F. Eastman, "Pyroelectric properties of $\mathrm{Al}(\mathrm{In}) \mathrm{GaN} / \mathrm{GaN}$ hetero- and quantum well structures," J. Phys.: Condens. Matter, vol. 14, p. 3399, 2002.

28. H. Morkoç, Handbook of Nitride Semiconductors and Devices, vol. 1. Wiley-VCH Verlag $\mathrm{GmbH}$, Weinheim, Germany, 2008.

29. Y.-N. Xu and W. Y. Ching, "Electronic, optical, and structural properties of some wurtzite crystals," Phys. Rev. B, vol. 48, p. 4335, 1993.

30. J. H. Ryou, Z. Lochner, and R. D. Dupuis, "Control of quantum-confined stark effect in InGaNbased quantum wells," IEEE J. Sel. Top. Quant. Electron., vol. 15, p. 1080, 2009.

31. H. P. Maruska and J. J. Tietjen, "The preparation and properties of vapor-deposited singlecrystal-line GaN," Appl. Phys. Lett., vol. 15, p. 327, 1969.

32. J. I. Pankove, H. P. Maruska, and J. E. Berkeyheiser, "Optical absorption of GaN," Appl. Phys. Lett., vol. 17, p. 197, 1970.

33. J. I. Pankove, E. A. Miller, D. Richman, and J. E. Berkeyheiser, "Electroluminescence in GaN," J. Lumin., vol. 4, p. 63, 1971.

34. L. Liu and J. H. Edgar, "Substrates for gallium nitride epitaxy," Mater. Sci. Eng. R, vol. 37, p. 61, 2002.

35. M. Ilegems, "Vapor epitaxy of gallium nitride," J. Cryst. Growth, vol. 13, p. 360, 1972.

36. W. Götz, L. T. Romano, J. Walker, N. M. Johnson, and R. J. Molnar, "Hall-effect analysis of GaN films grown by hydride vapor phase epitaxy," Appl. Phys. Lett., vol. 72, p. 1214, 1998.

37. R. Quay, Gallium Nitride Electronics. Springer-Verlag Berlin Heidelberg, 2008.

38. T. D. Moustakas, T. Lei, and R. J. Molnar, "Growth of GaN by ECR-assisted MBE," Physica B, vol. 185 , p. 36, 1993.

39. S. Nakamura, M. Senoh, and T. Mukai, "Highly P-Typed Mg-Doped GaN films grown with GaN buffer layers," Jpn. J. Appl. Phys., vol. 30, p. L1708, 1991.

40. J. A. V. Vechten, "Quantum dielectric theory of electronegativity in covalent systems. iii. pressure- temperature phase diagrams, heats of mixing, and distribution coefficients," Phys. Rev. $B$, vol. 7, p. 1479, 1972.

41. K. Harafuji, T. Tsuchiya, and K. Kawamura, "Molecular dynamics simulation for evaluating melting point of wurtzite type GaN crystal," J. Appl. Phys., vol. 96, p. 2501, 2004.

42. J. Karpiński, J. Jun, and S. Porowski, "Equilibrium pressure of $\mathrm{n}_{2}$ over GaN and high pressure solution growth of GaN," J. Cryst. Growth, vol. 66, p. 1, 1984. 
43. W. Utsumi, H. Saitoh, H. Kaneko, T. Watanuki, K. Aoki, and O. Shimomura, "Congruent melting of gallium nitride at $6 \mathrm{GPa}$ and its application to single-crystal growth," Nat. Mater., vol. 2, p. 735, 2003.

44. S. Porowski, "High pressure crystallization of III-V nitrides," Acta. Phys. Pol. A, vol. 87, p. 295, 1995.

45. S. Porowski, "Growth and properties of single crystalline GaN substrates and homoepitaxial layers," Mater. Sci. Eng. B, vol. 44, p. 407, 1997.

46. I. Grzegory, J. Jun, S. Krukowski, M. Boćkowski, and S. Porowski, "Crystal growth of iii-n compounds under high nitrogen pressure," Physica B, vol. 185, p. 99, 1993.

47. I. Grzegory, J. Jun, M. Boćkowski, S. Krukowski, M. Wróblewski, B. Łucznik, and S. Porowski, "III-V nitrides-thermodynamics and crystal growth at high $\mathrm{n}_{2}$ pressure," J. Phys. Chem. Solids, vol. 56, p. 639, 1995.

48. P. Perlin, I. Gorczyca, N. Christensen, I. Grzegory, H. Teisseyre, and T. Suski, "Pressure studies of gallium nitride: crystal growth and fundamental electronic properties," Phys. Rev. B, vol. 45, p. 13307, 1992.

49. R. Dwiliński, A. Wysmolek, J. Baranowski, M. Kamińska, R. Doradziński, J. Garczyński, and L. Sierzputowski, "GaN synthesis by ammonothermal method," Acta. Phys. Pol. A, vol. 88, p. 833, 1995.

50. R. Dwiliński, J. M. Baranowski, M. Kamińska, R. Doradziński, J. Garczyński, and L. Sierzputowski, "On GaN crystallization by ammonothermal method," Acta. Phys. Pol. A, vol. 90, p. 763, 1996.

51. J. W. Kolis, S. Wilcenski, and R. A. Laudise, "Material chemistry and bulk crystal growth of group iii nitrides in supercritical ammonia," Mater. Res. Soc. Symp. Proc., vol. 495, p. 367, 1998.

52. B. Raghothamachar, W. M. Vetter, M. Dudley, R. Dalmau, R. Schelesser, Z. Sitar, E. Michaels, and J. W. Kolis, "Synchrotron white beam topography characterization of physical vapor transport grown AlN and ammonothermal GaN," J. Cryst. Growth, vol. 246, p. 271, 2002.

53. R. Dwiliński, R. Doradziński, J. Garczyński, L. Sierzputowski, A. Puchalski, Y. Kanbara, K. Yagi, H. Minakuchi, and H. Hayashi, "Excellent crystallinity of truly bulk ammonothermal GaN," J. Cryst. Growth, vol. 310, p. 3911, 2008.

54. "Ammono." http://ammono.com/, Accessed: 2014-05-16.

55. H. Yamane, M. Shimada, S. J. Clarke, and F. J. DiSalvo, "Preparation of GaN single crystals using a na flux," Chem. Mater., vol. 9, p. 413, 1997.

56. M. Aoki, H. Yamane, M. Shimada, S. Sarayama, and F. J. DiSalvo, "GaN single crystal growth using high-purity na as a flux," J. Cryst. Growth, vol. 242, p. 70, 2002.

57. M. Aoki, H. Yamanea, M. Shimadaa, S. Sarayama, and F. J. DiSalvo, "Growth of $5 \mathrm{~mm} \mathrm{GaN}$ single crystals at $750^{\circ} \mathrm{c}$ from an Na-Ga melt," Cryst. Growth Des., vol. 1, p. 119, 2000. 
58. S. J. Pearton, J. C. Zolper, R. J. Shul, and F. Ren, "GaN: Processing, defects, and devices," J. Appl. Phys., vol. 86, p. 1, 1999.

59. S. Nakamura, S. J. Pearton, and G. Fasol, The Blue Laser Diode. Springer-Verlag Berlin Heidelberg GmbH, second ed., 2000.

60. S. J. Pearton, F. Ren, A. Zhang, and K. Lee, "Fabrication and performance of GaN electronic devices," Mater. Sci. Eng. R, vol. 30, p. 55, 2000.

61. C. Damian, "A new generation of gallium nitride $(\mathrm{GaN})$ based solid state power amplifiers for satellite communication.” Published by Advantech Wireless Inc., 2012.

62. G. D. Chen, M. Smith, J. Y. Lin, H. X. Jiang, and S. Wei, "Fundamental optical transitions in GaN," Appl. Phys. Lett., vol. 68, p. 2784, 1996.

63. S. Nakamura, T. Mukai, and M. Senoh, "High-power GaN P-N junction blue-light-emitting diodes," Jpn. J. Appl. Phys., vol. 30, p. L1998, 1991.

64. P. Gibart, B. Beaumont, and P. Vennégués, "Epitaxial lateral overgrowth of GaN," in Nitride Semiconductors (Handbook on Materials and Devices) (P. Ruterana, M. Albrecht, and J. Neugebauer, eds.), pp. 52-54, Wiley-VCH Verlag GmbH, Weinheim, Germany, 2003.

65. M. Nastasi and J. W. Mayer, Ion Implantation and Synthesis of Materials. Springer-Verlag Berlin Heidelberg, 2006.

66. G. F. Knoll, Radiation Detection and Measurement. John Wiley \& Sons, fourth ed., 2010.

67. J. F. Ziegler. SRIM-2013 software package, available online at http://www.srim.org.

68. K. E. Holbert, "Radiation effects and damage." http://holbert.faculty.asu.edu/ eee560/eee560 .html, 2007. Arizona State University, Accessed: 2014-06-03.

69. H. Zhu, R. S. Averback, and M. Nastasi, "Molecular dynamics simulations of a 10kev cascade in $\beta$-NiAl," Philos. Mag. A, vol. 71, p. 735, 1995.

70. P. D. Townsend, “Optical effects of ion implantation,” Rep. Prog. Phys., vol. 50, p. 501, 1987.

71. N. Tripathi and S. Rath, "Effect of thermal annealing and swift heavy ion irradiation on the optical properties of indium oxide thin films," J. Solid State Sci. Technol., vol. 3, p. 21, 2014.

72. A. Kumar, S. K. Tripathi, P. K. Kulriya, A. Tripathi, F. Singh, and D. K. Avasthi, "100 MeV Ag ions irradiation effects on the optical properties of $\mathrm{Ag}_{0.10}\left(\mathrm{Ge}_{0.20} \mathrm{Se}_{0.80}\right)_{0.90}$ thin films," J. Phys. D : Appl. Phys, vol. 43, p. 095302, 2010.

73. H. Bernas, Materials Science with Ion Beams. Springer-Verlag Berlin Heidelberg, 2010.

74. D. D. L. Rubia, "Irradiation-induced defect production in elemental metals and semiconductors: A review of recent molecular dynamics studies," Annu. Rev. Muter. Sci., vol. 26, p. 613, 1996.

75. J. L. Benton, S. Libertino, P. Kringhøj, D. J. Eaglesham, and J. M. Poate, "Evolution from point to extended defects in ion implanted silicon," J. Appl. Phys., vol. 82, p. 120, 1997. 
76. A. Pinos, S. Marcinkevičius, M. Usman, and A. Hallén, "Time-resolved luminescence studies of proton-implanted GaN," Applied Physics Letters, vol. 95, no. 11, p. 112108, 2009.

77. N. Xiang, F. Lin, H. P. Li, H. F. Liu, W. Liu, W. Ji, and S. J. Chua, "GaN-based semiconductor saturable absorber mirror operating around 415 nm," Thin Solid Films, vol. 515, p. 4484, 2007.

78. M. Yoshita, M. Kuramoto, M. Ikeda, and H. Yokoyama, "Mode locking of a GaInN semiconductor laser with an internal saturable absorber," Appl. Phys. Lett., vol. 94, p. 061104, 2009.

79. V. D. S. Dhaka, N. V. Tkachenko, H. L. E.-M. Pavelescu, S. Suomalainen, M. Pessa, K. Arstila, J. Keinonen, and K. Nordlund, "Ultrafast dynamics of $\mathrm{Ni}^{+}$-irradiated and annealed GaInAs/InP multiple quantum wells," Journal of Physics D: Applied physics, vol. 39, pp. 2659-2663, 2006.

80. V. D. S. Dhaka, N. V. Tkachenko, H. L. E.-M. Pavelescu, M. Guina, A. Tukiainen, J. Konttinen, M. Pessa, K. Arstila, J. Keinonen, and K. Nordlund, "Effects of heavy-ion and light-ion irradiation on the dynamics of InGaAs/GaAs quantum wells," Semiconductor science and technology, vol. 21, pp. 661-664, 2006.

81. T. Hakkarainen, E.-M. Pavelescu, K. Arstila, V. D. S. Dhaka, T. Hakulinen, R. Herda, J. Kontinen, N. Tkachenko, H. Lemmetyinen, J. Keinonen, and M. Pessa, "Optical properties of ion irradiated and annealed InGaAs/GaAs quantum wells and semiconductor saturable absorber mirrors," J. Phys. D: Appl. Phys., vol. 38, p. 985, 2005.

82. J. Mangeney, H. Chouman, G. Patriarche, G. Leroux, G. Aubin, J. C. Harmand, J. L. Oudar, and H. Bernas, "Comparison of light- and heavy-ion-irradiated quantum-wells for use as ultrafast saturable absorbers," Appl. Phys. Lett., vol. 79, p. 2722, 2001.

83. C. Björkas, K. Nordlund, K. Arstila, J. Keinonen, V. D. S. Dhaka, and M. Pessa, "Damage production in GaAs and GaAsN induced by light and heavy ions," J. Appl. Phys., vol. 100, p. $053516,2006$.

84. S. O. Kucheyev, J. S. Williams, A. I. Titov, G. Li, and C. Jagadish, "Effect of the density of collision cascades on implantation damage in GaN," Appl. Phys. Lett., vol. 78, p. 2694, 2001.

85. P. A. Karaseov, A. Y. Azarov, A. Titov, and S. Kucheyev, "Density of displacement cascades for cluster ions: An algorithm of calculation and the influence on damage formation in $\mathrm{ZnO}$ and GaN," Semiconductors, vol. 43, p. 691, 2009.

86. J. F. Ziegler. SRIM-2008.04 software package, available online at http://www.srim.org.

87. A. I. Titov, P. A. Karaseov, V. S. Belyakov, K. V. Karabeshkin, A. V. Arkhipov, S. O. Kucheyev, and A. Y. Azarov, "Molecular effect on surface topography of GaN bombarded with $\mathrm{PF}_{4}$ ions," Vacuum, vol. 86, pp. 1638-1641, 2012.

88. B. J. Alder and T. E. Wainwright, "Phase transition for a hard sphere system," J. Chem. Phys., vol. 27, p. 1208, 1957.

89. W. C. Swope, H. C. Andersen, P. H. Berens, and K. R. Wilson, "A computer simulation method for the calculation of equilibrium constants for the formation of physical clusters of molecules: Application to small water clusters," J. Chem. Phys., vol. 76, p. 637, 1982. 
90. C. W. Gear, "The numerical integration of ordinary differential equations of various orders." Report ANL 7126, 1966. Arizona National Laboratory.

91. C. W. Gear, Numerical initial value problems in ordinary differential equations. Prentice-Hall, Englewood Cliffs, New Jersey, 1971.

92. H. J. C. Berendsen, J. P. M. Postma, W. F. van Gunsteren, A. DiNola, and J. R. Haak, "Molecular dynamics with coupling to external bath," J. Chem. Phys., vol. 81, no. 8, p. 3684, 1984.

93. M. Born and R. Oppenheimer, "Zur quantentheorie der molekeln (on the quantum theory of molecules)," Annalen der Physik, vol. 389, p. 457, 1927.

94. D. Brenner, "Relationship between the embedded-atom method and tersoff potentials," Phys. Rev. Lett, vol. 63, p. 1022, 1989.

95. J. Tersoff, "Chemical order in amorphous silicon carbide," Phys. Rev. B, vol. 49, p. 16349, 1994.

96. D. Brenner, "Empirical potential for hydrocarbons for use in simulating the chemical vapor deposition of diamond films," Phys. Rev. B, vol. 42, p. 9458, 1990.

97. J. Nord, K. Albe, P. Erhart, and K. Nordlund, "Modelling of compound semiconductors: analytical bond-order potential for gallium, nitrogen and gallium nitride," J. Phys.: Condens. Matter, vol. 15, p. 5649, 2003.

98. J. F. Ziegler, J. P. Biersack, and U. Littmark, The Stopping and Range of Ions in Matter. New York: Pergamon, 1985.

99. S. Saito, Y. Endo, and E. Hirota, "The microwave spectrum of the $\mathrm{PF}_{2}$ radical in the $\tilde{\mathrm{X}}^{2} \mathrm{~B}_{1}$ ground vibronic state," J. Chem. Phys, vol. 85, p. 1778, 1986.

100. G. S. Tschumper, J. T. Fermann, and H. F. S. III, "Structures, thermochemistry, and electron affinities of the $\mathrm{PF}_{n}$ and $\mathrm{PF}_{n}^{-}$series, n=1-6," J. Chem. Phys, vol. 104, p. 3676, 1996.

101. F. M. Tao, "On the use of bond functions in molecular calculations," J. Chem. Phys, vol. 98, p. 2481, 1993.

102. J. Nord, K. Nordlund, and J. Keinonen, "A molecular dynamics study of damage accumulation in GaN during ion beam irradiation," Phys. Rev. B, vol. 68, p. 184104, 2003.

103. C. Pryor, J. Kim, L. W. Wang, A. J. Williamson, and A. Zunger, "Comparison of two methods for describing the strain profiles in quantum dots," J. Appl. Phys., vol. 83, no. 5, p. 2548, 1998.

104. H. H. Tan, J. S. Williams, J. Zou, D. J. H. Cockayne, S. J. Pearton, and R. A. Stall, "Damage to epitaxial GaN layers by silicon implantation,” Appl. Phys. Lett., vol. 69, p. 2364, 1996.

105. H. H. Tan, C. Jagadish, J. S. Williams, J. Zou, and D. J. H. Cockayne, "Ion damage buildup and amorphization processes in GaAs- $\mathrm{Al}_{x} \mathrm{Ga}_{1-x}$ As multilayers," Appl. Phys. Lett., vol. 80, p. 2691, 1996.

106. C. Björkas and K. Nordlund, "Comparative study of cascade damage in fe simulated with recent potentials," Nucl. Instr. Meth. Phys. Res. B, vol. 259, p. 853, 2007. 
107. A. Y. Polyakov, S. J. Pearton, P. Frenzer, F. Ren, L. Liu, and J. Kim, "Radiation effects in GaN materials and devices," J. Mater. Chem. C, vol. 1, pp. 877-887, 2013.

108. Y. Nakanishi, A. Wakahara, H. Okada, A. Yoshida, T. Ohshima, and H. Itoh, "Effect of 3 mev electron irradiation on the photoluminescence properties of Eu-doped GaN," Appl. Phys. Lett., vol. 81, p. 1943, 2002.

109. J. W. Tringe, T. E. Felter, C. E. Talley, J. D. Morse, C. G. Stevens, J. M. Castelaz, and C. Wetzel, "Radiation damage mechanisms for luminescence in Eu-doped GaN," J. Appl. Phys., vol. 101, p. $054902,2007$.

110. S. Li and A. Waag, "GaN based nanorods for solid state lighting," J. Appl. Phys., vol. 111, p. 071101, 2012.

111. C. Ronning, E. P. Carlson, and R. F. Davis, "Ion implantation into gallium nitride," Physics Reports, vol. 351, p. 349, 2001.

112. K. Lorenz, E. Nogales, S. M. C. Miranda, N. Franco, B. Méndez, E. Alves, G. Tourbot, and B. Daudin, "Enhanced red emission from praseodymium-doped gan nanowires by defect engineering," Acta Materialia, vol. 61, p. 3278, 2013.

113. J. Rodrigues, S. M. C. Miranda, A. J. S. Fernandes, E. Nogales, L. C. Alves, E. Alves, G. Tourbot, T. Auzelle, B. Daudin, B. Méndez, T. Trindade, K. Lorenz, F. M. Costa, , and T. Monteiro, "Towards the understanding of the intentionally induced yellow luminescence in GaN nanowires," Phys. Status Solidi C, vol. 10, p. 667, 2013.

114. I. Shalish, L. Kronik, G. Segal, Y. Rosenwaks, Y. Shapira, U. Tisch, and J. Salzman, "Yellow luminescence and related deep levels in unintentionally doped GaN films," Phys. Rev. B, vol. 59, p. $9748,1999$.

115. M. A. Reshchikov, H. Morkoç, S. S. Park, and K. Y. Lee, "Yellow and green luminescence in a freestanding GaN template," Appl.Phys. Lett., vol. 78, p. 3041, 2001.

116. Q. Li and G. T. Wang, "Spatial distribution of defect luminescence in GaN nanowires," Nano Lett., vol. 10, p. 1554, 2010.

117. W. Ren, A. Kuronen, and K. Nordlund, "Molecular dynamics of irradiation-induced defect production in GaN nanowires," Phys. Rev. B, vol. 86, p. 104114, 2012. 